\title{
Energy Efficient Hybrid Satellite Terrestrial 5G Networks with Software Defined Features
}

\author{
Jiaxin Zhang, Xing Zhang, Muhammad Ali Imran, Barry Evans, Yan Zhang, and Wenbo Wang
}

\begin{abstract}
In order to improve the manageability and adaptability of future $5 \mathrm{G}$ wireless networks, the software orchestration mechanism, named software defined networking (SDN) with Control and User plane (C/U-plane) decoupling, has become one of the most promising key techniques. Based on these features, the hybrid satellite terrestrial network is expected to support flexible and customized resource scheduling for both massive machinetype-communication (MTC) and high-quality multimedia requests while achieving broader global coverage, larger capacity and lower power consumption. In this paper, an end-to-end hybrid satellite terrestrial network is proposed and the performance metrics, e. g., coverage probability, spectral and energy efficiency (SE and EE), are analysed in both sparse networks and ultra-dense networks. The fundamental relationship between $\mathrm{SE}$ and $\mathrm{EE}$ is investigated, considering the overhead costs, fronthaul of the gateway (GW), density of small cells (SCs) and multiple quality-ofservice $(Q 0 S)$ requirements. Numerical results show that compared with current LTE networks, the hybrid system with $\mathrm{C} / \mathrm{U}$ split can achieve approximately $40 \%$ and $80 \% \mathrm{EE}$ improvement in sparse and ultra-dense networks respectively, and greatly enhance the coverage. Various resource management schemes, bandwidth allocation methods, and on-off approaches are compared, and the applications of the satellite in future 5G networks with software defined features are proposed.
\end{abstract}

Index Terms: Hybrid satellite terrestrial networks, software defined networks, spectral and energy efficiency, coverage probability, on-off strategy, sparse and ultra-dense networks, 5G.

\section{INTRODUCTION}

$\mathrm{W}$ ITH the explosive growth of high-data-rate multimedia and machine-type-communication services, next gener-

Manuscript received March 17, 2016; approved for publication by Zhu Han, Editor, November 1, 2016.

This work is supported by the National Science Foundation of China (NSFC) under grant 61372114,61571054 and 61631005 , by the New Star in Science and Technology of Beijing Municipal Science \& Technology Commission (Beijing Nova Program: Z151100000315077), by the projects 240079/F20 funded by the Research Council of Norway, by the project IoTSec - Security in IoT for Smart Grids, with number 248113/O70 part of the IKTPLUSS program funded by the Norwegian Research Council.

J. Zhang, X. Zhang and W. Wang are with are with the School of Information and Communication Engineering, Beijing University of Posts and Telecommunications, Beijing, 100876, China. X. Zhang is also with Beijing Advanced Innovation Center for Future Internet Technology Beijing University of Technology (BJUT), Beijing, China. X. Zhang is the corresponding author (zhangx@ieee.org).

M. A. Imran is with the School of Engineering, University of Glasgow, UK (email: muhammad.imran@glasgow.ac.uk).

B. Evans is with the Institute for Communication Systems (ICS), University of Surrey, Guildford, UK (email: b.evans@ surrey.ac.uk).

Y. Zhang is with the Simula Research Laboratory and University of Oslo, Norway (email: yanzhang@ieee.org).

Part of this work has been published in a workshop in CAMAD and VTC.

Digital Object Identifier 10.1109/JCN.2015.000074 ation $(5 \mathrm{G})$ networks have attracted much attention from both academic research and commercial exploitation in the information and communications technology (ICT) field to enable highly efficient, ultra-reliable, dependable, secure, privacy preserving and delay critical services. It is expected that 1000 times or greater traffic requirements will be generated by 2020, achieving rates of $1 \mathrm{Gbps}$ per user equipment (UE) with $10 \mathrm{Gbps}$ peak speed [1] [2]. As a result, the densification of SCs has become a trend to improve the area spectral efficiency, reaching more than 1000 small cells per $\mathrm{km}^{2}$ with a radius of even less than 10 meters per cell [3] [4]. The current use of ICT accounts for 5.7 percent of global electricity consumption and 1.8 percent of global carbon emissions, resulting in approximately $\$ 10$ billion cost for mobile network operators worldwide per year [5]. Green communications, with energy and spectral efficiency trade-off, is a key indicator for $5 \mathrm{G}$ wireless systems, and has been one of the main topics to be studied and discussed for future $5 \mathrm{G}$ wireless networks [6].

$5 \mathrm{G}$ services will place very stringent requirements in terms of achievable coverage, data rates, latency, reliability, and energy consumption, which may not all be met at the same time by one architecture. On the one hand, the widespread requirements of high-quality mobile multimedia services, requiring a single connection with continuous high rate video frame delivery, are one of the key objectives of the future wireless networks. On the other hand, with the Internet of Things (IoT) connected intelligently in future smart cities [7], new applications of machine-tomachine (M2M) communications are quite different from highdefinition video streaming in the former case [8]. For example, massive access machine type communication services require huge numbers of connections, bursty type traffic, low or zero mobility and a very small amount of downlink data per device (e.g., only a few bits of information per transaction with long inter-arrival periods) [9]. These MTC requirements with smallsized persistent bursty traffic services will provide a heavy load on the wireless access and core networks, lower the probability for SCs to be turned off and waste bandwidth resources.

In order to address these challenges and improve the manageability and adaptability of networks, thus achieving the concept of "multiple architectures adapted to each service", the software orchestration mechanism with control and user plane separation has attracted considerable attention [10]. The 3rd Generation Partnership Project (3GPP) standards have created a specific study group on small cell enhancement in Release 12, focusing on the architecture design based on the SDN concept [11].

According to the idea of control and user plane separation, effort is made to improve the efficiency of the network and provide on-demand services to various application requirements based on the user behavior [12] utilizing the wireless big data 
[13]. The "always-on" Radio Resource Control (RRC) control signalling in M2M type communications and the low-data-rate services can be provided by a macro cell for the control plane. Meanwhile, the on-demand high-data-rate requests can be satisfied by the small cells. Various architecture concepts for future $5 \mathrm{G}$ wireless networks based on software defined features have been proposed from academic research and industrial corporations. The GreenTouch consortium gives an outline of "Beyond Cellular Green Generation (BCG2)" project and illustrates the benefits of small cells in a sleeping strategy [14]. Huawei proposes a two-layer network functionality separation scheme by taking UE states, the network functionality and signals into consideration, hence targeting a low control signaling overhead and flexible network reconfiguration for future mobile networks [15]. In [10], the author advances the idea of pure dataonly carriers to remove most of the public control signalling to the control plane and various procedures are designed from the aspects of high energy efficiency and lower interference from signalling. All of these contributions promote the flexibility and programmability, dynamic reconfiguration and dynamic resource allocation to provide a more flexible network.

Although the $\mathrm{C} / \mathrm{U}$ split concept is well-known and studied by both industry and academia, this has been contributed to terrestrial networks. However, satellite communications have significant advantages including world-wide coverage, efficien$\mathrm{t}$ content distribution, providing resilience and energy saving [16], so that the integration of satellite and terrestrial networks could become an important feature for 5G. The European Union has set up the 5G Public Private Partnership (5GPPP) research programme to fund research from industry, academia and research organizations toward an integrated 5G standard [17]. Even the well-known social-network Facebook has released detailed plans on how company is exploring ways to use aircraft and satellites to beam internet access down into communities from the sky [18]. Satellite communication systems with a global distribution of terminals and base stations, including the context information and user behaviors, can be utilized to realize an information-centric network (ICN) with a more flexible delivery strategy and dynamic bandwidth allocation scheme [19]. Integration of the satellite to provide wide-area sparse coverage with cellular providing dense local coverage seems to be attractive.In such a hybrid architecture, signalling could be provided via the satellite [20] [21][22]. However, what is the role of satellite in the $\mathrm{C} / \mathrm{U}$ split networks in future $5 \mathrm{G}$ systems? Will the satellite be able to be integrated to future networks in an efficient and green network? To the best of our knowledge, the performance and application analysis of satellites under $\mathrm{C} / \mathrm{U}$ split in hybrid satellite terrestrial networks is still an open issue.

In this paper, an end-to-end satellite terrestrial network with software defined features is proposed under two typical future application scenarios: sparse and ultra-dense networks. In the sparse network scenario, we pay more attention to the overall performance of the system, and in the ultra-dense network, the users with different QoS constraints are considered: primary user equipment (PUE) with MTC-type of service and secondary user equipment (SUE) with relative high data-rates. The key performance metrics are studied considering related factors, e. g., the density of SCs, and the overhead cost, etc. In addition, re- source management schemes and bandwidth allocation methods are proposed and compared with current LTE networks. Furthermore, on-off approaches of SCs are proposed and analysed from the perspective of coverage and energy efficiency. It is shown that compared with the current LTE network, the hybrid system with C/U split can achieve nearly $40 \%$ EE improvement and greatly enhance the U-plane coverage in sparse networks. In ultra-dense networks, the benefits of the on-off strategy in the hybrid network can increase the EE by approximately $80 \%$ while also providing better coverage. In the end, main applications of the hybrid network are proposed, with different resource management schemes, bandwidth allocation methods and on-off energy saving approaches suggested under different deployment scenarios.

The main contributions are summarized as follows:

- An end-to-end hybrid satellite-terrestrial architecture with software defined features is proposed. The overhead cost for both the LTE and the hybrid system is evaluated. Both sparse and ultra-dense scenarios for future $5 \mathrm{G}$ networks are suggested with different types of users with various data-rate and delay requirements.

- Based on the use of a stochastic geometry tool, key performance indicators are analysed, including coverage probability, throughput, energy consumption, SE and EE. Fundamental relationships are derived between the performance and key factors, including overhead cost, density of SCs, delay, transmission and circuit power, gateway fronthaul, and multiple qualityof-service (QoS) requirements.

- Resource management schemes, bandwidth allocation methods and on-off approaches are proposed and comparisons are made with LTE networks. Applications of satellites are summarised for both sparse and ultra-dense networks respectively.

Our results and analysis provide key insights into the deployment of hybrid satellite terrestrial networks which are seen as a promising solution for future heterogeneous wireless network, aiming at enabling research directions for the hybrid satelliteterrestrial system.

The reminder of the paper is organized as follows. Section II proposes an end-to-end hybrid satellite terrestrial architecture in sparse and ultra-dense networks and the roles of satellite, SCs and gateways in $\mathrm{C} / \mathrm{U}$ split networks are introduced. In Section III, the deployment model, path loss and fading channel model in terrestrial and satellite networks, access strategy, resource management schemes, bandwidth allocation methods, and SC "on-off" approaches are given as a foundation for theoretical analysis. In Section IV and V, multiple main performance indicators are analysed based on the stochastic geometry in sparse and ultra-dense scenarios respectively. Numerical results are illustrated from various scenarios and comparisons are made between various hybrid network schemes and current LTE systems in Section VI. Conclusions, including main application scenarios and appropriate architectures, are suggested and future work is discussed for $5 \mathrm{G}$ green hybrid satellite terrestrial networks.

\section{Our Proposed Hybrid Satellite Terrestrial Architecture}

In this section, end-to-end hybrid satellite terrestrial networks with software defined features are proposed in both sparse and ultra-dense networks, as shown in Fig. 1. In the C/U split net- 


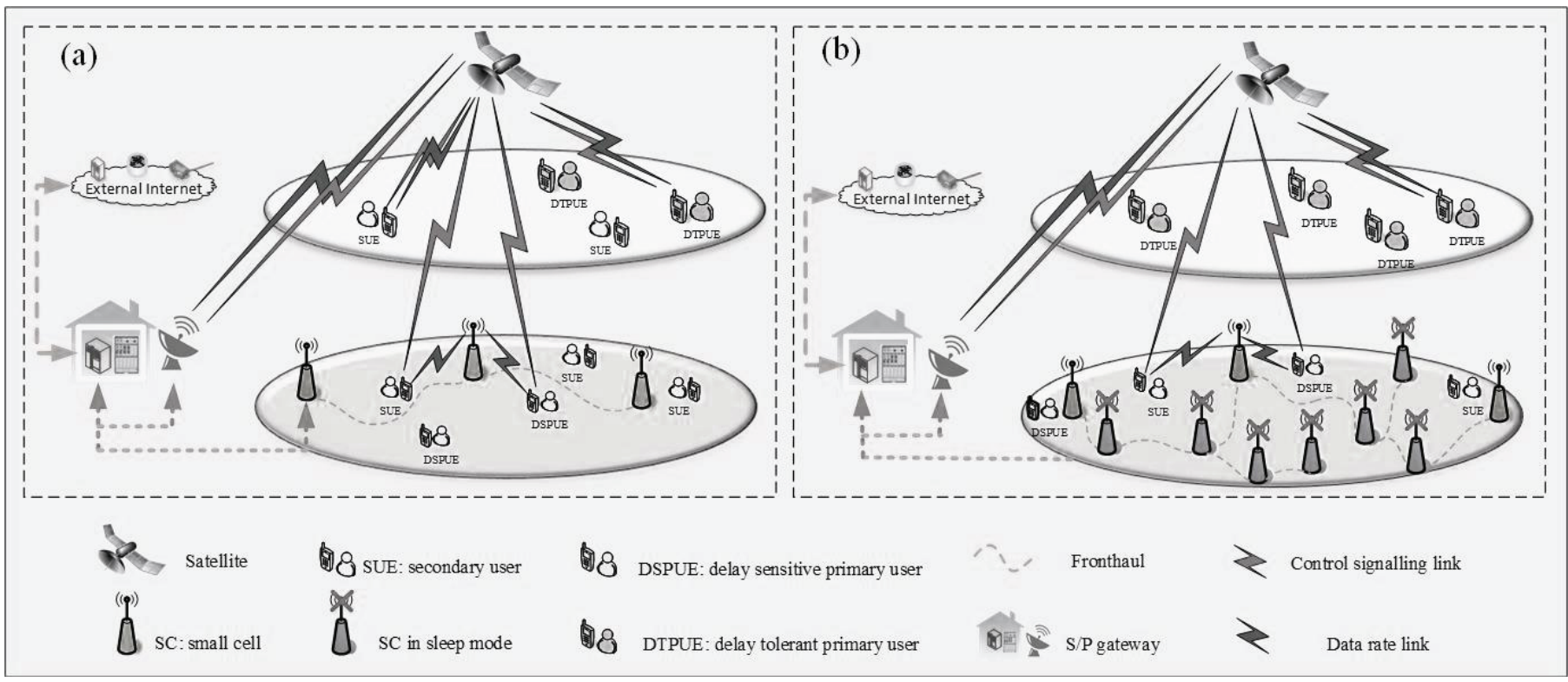

Fig. 1. End-to-end hybrid satellite terrestrial networks with control and user plane separation: (a) Sparse networks; (b) Ultra-dense networks.

work, the system becomes more flexible and adaptive to various requests, according to the data-rate or the delay requirements. The slave radio resource management (RRM) function in the small cell can be configured under the central control of masterRRM in the satellite. So that the access strategy can be used with different resource management schemes, bandwidth allocation methods and on-off approaches. In sparse networks, there are few small cells in the coverage of the satellite cell to provide low-rate services, e.g., remote sensors, interactive data services, emergency and E-health systems, which are the most importan$\mathrm{t}$ scenarios for satellite communication systems. In ultra-dense scenarios, thousands of SCs are deployed within a certain area, resulting in serious interference, huge energy consumption and possibility of handover failure. Thus the satellite can help to enhance the coverage and increase the probability for SCs to go into sleep mode. In addition, the users are divided into two types: PUE with machine-type communication and SUE with relatively high data-rate requirement. The typical message size of the PUE is generally very short, e.g, 200 bytes or even just a few bits to inform of the existence or absence of a given even$t$ [9] [23], while the total number of PUEs could be orders of magnitude greater than the number of SUEs in future systems.

Within these architectures, the satellite is assumed as the home subscriber server (HSS) with a central database containing information about the network's subscribers and mobility management entity (MME) with signalling functions related to the mobility and security of the Evolved Universal Terrestrial Radio Access Network (E-UTRAN) access. In addition, the satellite maintains seamless large-scale coverage in the C-plane and also for low rate data transmissions, e.g., MTC service, remote sensing, environment monitoring, in the U-plane for delaytolerant PUEs. SC coverage will use high frequency (e.g., initially less than $6 \mathrm{GHz}$ but eventually millimetre waves), and secondary users (SUEs) and delay-sensitive PUEs also retain the C-plane link with the satellite whilst receiving high-quality mobile multimedia transmission from the SCs in the terrestrial networks in the U-plane, so that the control channel and public signalling of the SCs can be significantly simplified. In such a hybrid network, the satellite provides the C-plane and the terrestrial part is responsible for the actual data transfer in the Uplane. It has been shown that the signalling overhead and public control channel in the downlink costs in small cells in LTE and hybrid networks are nearly $28 \%$ [24] and $15 \%$ respectively [20]. However, the satellite is assumed to have limited computing ability, so that it is more realistic for the S/P-GW to process the related information and send it back over the satellite. Furthermore, the uplinks may have extremely poor performance, so the gateway is utilized. The traffic in terrestrial networks is routed and aggregated in the gateway, including the C-plane control signalling and U-plane traffic, and then sent back via the satellite to the external internet.

Satellites operating in $\mathrm{S}$ band $(2-3 \mathrm{GHz})$ are more suitable for the integrated UE as the frequencies are closer. We also consider constellations of LEO satellites (altitude of around $1000 \mathrm{~km}$ ) as the delay is reduced and the UE power requirements are lower. In the future, it may be possible to use the constellations of high throughput satellite (HTS) in Ka or Ku band [25], but these are not considered herein. The LEO satellites employ a large number of spot-beams (satellite cells) within their coverage as well as frequency re-use between them [26]. So co-channel interference is an issue but we consider that it is not a dominant parameter herein. The satellite spot beam handover will be designed into the constellation systems and need not be considered here. Doppler shift is also compensatable and is not considered. The latency issue drives us to consider LEO satellites and its affect on the C-plane signallings is considered in other papers.

\section{System Model}

This section provides the system model for our proposed hybrid satellite terrestrial networks as shown in Fig. 1. The resource management schemes, bandwidth allocation methods and "on-off" approaches are summarized in Table 1. 
Table 1. System Configuration.

\begin{tabular}{|c|c|c|}
\hline \multirow{2}{*}{ Channel Model } & Small Cells & Rayleigh Fading \\
\hline & Satellite & Land Mobile Satellite (LMS) Channel \\
\hline \multirow{4}{*}{ Sparse Networks } & Access Strategy & Open Access based on SINR \\
\hline & Coverage Probability & SINR Coverage Probability \\
\hline & \multirow{2}{*}{ Resource Management Schemes } & Centralized Resource Management Schemes (CRMS) \\
\hline & & Distributed Resource Management Schemes (DRMS) \\
\hline \multirow{8}{*}{ Ultra-dense Networks } & Access Strategy & Closed Access based on the User Type \\
\hline & Coverage Probability & Service Coverage Probability \\
\hline & \multirow{2}{*}{ Resource Management Schemes } & Centralized Resource Management Schemes (CRMS) \\
\hline & & Distributed Resource Management Schemes (DRMS) \\
\hline & \multirow{2}{*}{ Bandwidth Allocation Methods } & User Number Based allocation Scheme (NBS) \\
\hline & & User Requirement Based allocation Scheme (RBS) \\
\hline & \multirow{2}{*}{ On-off Approaches } & Random Sleep Mode (RSM) \\
\hline & & Traffic Based Mode (TBM) \\
\hline
\end{tabular}

\section{A. Deployment Model}

In the satellite spot beam coverage, the SCs are deployed as the classical homogeneous Spatial Poisson Point Processes (PP$\mathrm{P})$ distribution, which is a widely used model with tractable characteristics to model the random effect of user and base station deployment in the cellular network [27]. The density of SCs in this distribution $\Phi$ is $\lambda_{\mathrm{b}}$, which is the number of small cells in the coverage of the satellite narrow spot beam. The constant transmission power and bandwidth of satellite and small cells are $P_{t s}, P_{t b}, W_{s}, W_{b}$ respectively. Assume that the nearest distance from the user to the SCs is $r$, the received power from the SC can be modeled as $P_{t b} h_{t b} r^{-\alpha}$, where the standard power loss propagation model is used with path loss exponent $\alpha$ and i.d.d. Rayleigh fading on all links from the small cell are modeled as exponential distributions with mean $1 / u: h_{t b} \sim \exp (u)$. Taking advantage of the PPP properties, the distribution of $r$ can be derived as follows:

$$
f_{r}(r)=e^{-\pi \lambda_{b} r^{2}} 2 \pi \lambda_{b} r .
$$

The users are deployed homogeneously as an SPPP distribution in this scenario, and the density of primary users and secondary users are $\lambda_{P U E}$ and $\lambda_{S U E}$ respectively.

\section{B. Pathloss and Fading Model}

The land mobile satellite (LMS) channel model is used for modeling the satellite radio channel, which is widely used in the satellite networks [28] [29]. The probability density function (PDF) $f_{\gamma_{s d}}(\gamma)$ of the instantaneous received signal-to-noise ratio (SNR) $\gamma$ from the satellite to the destination is given in [28] as

$$
\begin{aligned}
f_{\gamma_{s d}}(\gamma)= & \frac{\Omega}{2 b_{0} \overline{\gamma_{s d}}}\left(\frac{2 b_{0} m}{2 b_{0} m+\Omega}\right)^{m} \exp \left(-\frac{\Omega \gamma}{2 b_{0} \overline{\gamma_{s d}}}\right) \\
& { }_{1} F_{1}\left(m, 1, \frac{\Omega^{2} \gamma}{2 b_{0} \overline{\gamma_{s d}}\left(2 b_{0} m+\Omega\right)}\right),
\end{aligned}
$$

where $\Omega$ is the average power of line-of-sight (LOS) componen$\mathrm{t}, 2 b_{0}$ is the average power of the multi-path component and $m$ is the Nakagami parameter ranging from 0 to $\infty$. When $m=0$ and $m=\infty$, the PDF of $\gamma_{s d}$ follows Reyleigh and Rice distributions respectively. Typically in this paper, the deep shadowing channel is adopted with the LMS channel parameters according to [30], where $b_{0}=0.0158, m=2.56$ and $\Omega$ is 0.123 . The function ${ }_{1} F_{1}(., .,$.$) is the confluent hyper-geometric function:$

$$
{ }_{1} F_{1}(a, b, c)=\sum_{n=0}^{\infty} \frac{a^{(n)}}{b^{(n)} n !} z^{n},
$$

where $x^{(n)}=x(x+1) \ldots(x+n+1)$. The average received transmission SNR $\overline{\gamma_{s d}}$ of UE is

$$
\begin{gathered}
\overline{\gamma_{s d}}=\frac{\mathrm{P}_{\mathrm{rs}}}{N_{d}}, \\
\mathrm{P}_{\mathrm{rs}}=\frac{P_{t s} G_{t} G_{r}}{L}\left(\frac{\lambda}{4 \pi d}\right)^{2}, \\
N_{d}=k T_{t} \cdot W_{s},
\end{gathered}
$$

where $\mathrm{P}_{\mathrm{rs}}$ is the received power including path loss and $N_{d}$ is the noise power at the terminal. For the LEO system, $\lambda$ is the wavelength, $d$ is the altitude of the LEO, $L$ is the atmosphere loss, and $G_{t}, G_{r}$ are the typical antenna gains of transmitter$\mathrm{s}$ and receivers in the downlink. The thermal noise $N_{d}$ should be taken into consideration because there is no interference between satellite and small cells, so that the satellite network is not an interference limited network. $k$ is the Blotzmann constant $1.38 \times 10^{-23} \mathrm{~J} / \mathrm{K}, T_{t}$ is the noise temperature of terminal and $W_{s}$ is the bandwidth of satellite.

\section{Access Strategy}

In this paper, there are two main scenarios, sparse and ultradense networks, as shown in Table 1, where access strategies vary based on the different scenarios.

In sparse networks, as the density of UEs and SCs are relatively much lower, we pay more attention to the overall Signal-toInterference-plus-Noise Ratio (SINR) coverage and the overall throughput of the whole system. Under the C/U split architecture, all of the UEs have C-plane connection via satellite, while the U-plane access strategy is open access, based on the Reference Signal Receiving Power (RSRP). The SCs are configured with bias $\theta$, which can be used to adjust the probability of getting U-plane access to satellite or SCs. The access strategy in 
the U-plane is given as follows :

$$
\left\{\begin{array}{c}
\theta \frac{P_{\mathrm{tb}} E\left[h_{t b}\right]}{r^{\alpha}}>\mathrm{P}_{r s}, \text { get access to the } S C \\
\theta \frac{P_{\mathrm{tb}} E\left[h_{t b}\right]}{r^{\alpha}}<\mathrm{P}_{r s}, \text { get access to the satellite }
\end{array},\right.
$$

where $\frac{P_{\mathrm{tb}} E\left[h_{t b}\right]}{r^{\alpha}}$ is the RSRP in the terrestrial networks. Then we suggest substitution of $\eta=\sqrt[\alpha]{\frac{\theta P_{\mathrm{tb}}}{u \mathrm{P}_{r s}}}$, deriving (7) as

$$
\left\{\begin{array}{c}
r<\eta, \text { get access to the } S C \\
r>\eta, \text { get access to the satellite }
\end{array} .\right.
$$

However, in ultra-dense networks, as the small cells can support quite large data-rates and much better SINR coverage, the QoS of each user will be of significant importance and the access strategy is also based on the rate and delay requirements of a typical user. Thus the closed access strategy is used, where the SUE and delay-sensitive PUE obtain access to the small cells and delay-tolerant PUEs are supported by the satellite.

\section{Resource Management Schemes}

To determine the best way to utilize satellites in future $5 \mathrm{G}$ networks, the role of the satellite in hybrid networks with software defined features are compared in this paper with different resource management schemes, which can be categorized as follows:

1. The LTE system (LTE): both the C-plane and U-plane of UEs have access to small cells

2. Distributed Resource Management Schemes (DRMS): all of the U-plane traffic required from UEs are routed from the core network to small cells directly by the gateway, and the satellite only provides $\mathrm{C}$-plane coverage and RRC-connection mobility control information.

3. Centralized Resource Management Schemes (CRMS): the gateway having computing and storage capability, operates as HSS and MME, taking charge of the central resource allocation strategy by adjusting the bias $\theta$ of probability for users to get access to the satellite and SCs. In this way, the satellite cooperates with the small cells in the U-plane under the central control of the gateway and the traffic can be offloaded onto the satellite.

In Section IV and V, these schemes are presented theoretically and compared for better understanding of the role of the satellite in the $\mathrm{C} / \mathrm{U}$ split hybrid architecture.

\section{E. Bandwidth Allocation Methods}

In ultra-dense networks, the bandwidth of the small cells has to be allocated to both SUE and delay-tolerant PUE. Two bandwidth allocation schemes are considered in this paper:

1. NBS: user Number Based allocation Scheme: the bandwidth is allocated based on the number of SUEs and delay-tolerant PUEs, so more users can enjoy greater bandwidth, which is fairer to all of the users;

2. RBS: user Requirement Based allocation Scheme: the bandwidth is allocated based on the traffic requirement of SUEs and delay tolerant PUEs, so that the QoS of UE is also involved in this scheme.

\section{F. On-off Approaches}

In ultra-dense networks, with the satellite providing the whole coverage for the C-plane, the small cells have a greater probability to go into sleep mode. Two on-off approaches are discussed in this paper in Section V:

1. Random Sleep Mode (RSM): each SC is turned off with equivalent probability $\varsigma$;

2. Traffic Based Mode (TBM): the small cells with no active users attached go into sleep mode, whereas the other SCs remain active.

In the following two sections, under the defined hybrid system, various strategies are analysed and their related performance results are illustrated in Section VI.

\section{Sparse Networks Analysis}

In this section, the distributed and centralized schemes (DRMS and CRMS) are compared and efficient resource management mechanisms suggested for the hybrid networks.

In sparse networks, the coverage probability is defined as the SINR coverage probability that a randomly chosen user can achieve a targeted SINR threshold. An open access policy is employed in the sparse network, so that the access strategy is taken into consideration. As we focus on the overall performance of the whole hybrid system instead of a specific user, user-level differences are ignored in this section and only the large scale path loss in equation (5) is considered in the satellite networks, where the fading and multi-path effects play an insignificant role.

\section{A. Coverage Probability}

Definition 1 (SINR Coverage) The Signal-to-interference-plusnoise Ratio (SINR) coverage probability is the probability that a randomly chosen user can achieve a targeted SINR $T$ :

$$
P_{\mathrm{cov}}=E_{r}\{P[S I N R(r)>T]\} .
$$

This definition is equivalent to the probability of the fraction of users in the networks that have achieved the corresponding threshold. Assuming that the path loss exponent is 4 and the noise is neglected, based on the stochastic geometry knowledge [27], with the access strategy in (8), the U-plane coverage probability for the two strategies can be obtained:

- Coverage Probability in LTE

$$
P_{\text {cov } \_L T E}=\frac{1}{1+\sqrt{T}\left(\frac{\pi}{2}-\arctan \left(\frac{1}{\sqrt{T}}\right)\right)},
$$

- Coverage Probability in hybrid networks with DRMS

$$
P_{\text {Cov } \_D R M S}=\frac{1}{1+\sqrt{T}\left(\frac{\pi}{2}-\arctan \left(\frac{1}{\sqrt{T}}\right)\right)},
$$

- Coverage Probability in hybrid networks with CRMS

$$
\begin{aligned}
& P_{\text {cov_CRMS }}=P_{\text {COv_SC }}+P_{\text {COv_LEO }} \\
& \left.=\mathrm{E}_{r}\left(P\left[S I N R_{b}>T \mid r\right]\right) \mathrm{P}(r<\eta)\right\} \\
& \left.+\mathrm{E}_{r}\left(P\left[S I N R_{s}>T \mid r\right]\right) \mathrm{P}(r>\eta)\right\} \\
& =\int_{0}^{\pi \lambda_{b}} \sqrt{\frac{P_{t b} \theta(4 \pi \mathrm{d})^{2} L}{P_{t s} \lambda^{2} G_{t} G_{r}}} e^{-v\left(1+\sqrt{T}\left(\frac{\pi}{2}-\arctan (1 / \sqrt{T})\right)\right)} d v \\
& +e^{-\pi \lambda_{b} \sqrt{\frac{P_{t b}}{P_{t s}} \cdot \frac{\theta}{\lambda^{2}} \cdot \frac{(4 \pi d)^{2} L}{G_{t} G_{r}}}} 1\left(\frac{P_{t s} G_{t} G_{r} \lambda^{2}}{(4 \pi d)^{2} L \sigma^{2}}>T\right),
\end{aligned}
$$


which is the sum of the coverage probability of SC $P_{\text {cov }} S C$ and the coverage probability of LEO satellite $P_{\text {cov_LEO }}$. Here the function 1(A) denotes the indicator of event $\mathrm{A}$.

The SINR coverage of LTE and DRMS in hybrid networks are the same, because the U-plane is only supported by small cells. However, the coverage in CRMS strategies is also influenced by the satellite with the factors related to the access strategy, e.g., the density of small cells, the transmission power and the loss, affecting the coverage performance. With the density of small cell $\lambda_{b} \rightarrow \infty,\left(e^{-\pi \lambda_{b} \sqrt{\frac{P_{t b}}{P_{t s}} \cdot \frac{\theta}{\lambda^{2}} \cdot \frac{(4 \pi d)^{2} L}{G_{t} G_{r}}}}\right) \rightarrow 0$, so that the

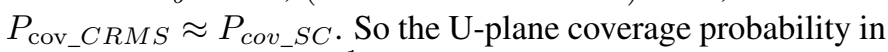
(12) comes to $\frac{1}{1+\sqrt{T}\left(\frac{\pi}{2}-\arctan \left(\frac{1}{\sqrt{T}}\right)\right)}$.

\section{B. Throughput}

\section{B.1 LTE}

The throughput of LTE networks under this scenario has been studied in [27] and is used as the baseline for comparison. The mean data rate of the downlink is around 2.15bps $/ \mathrm{Hz}$ and the overhead cost is $O_{\text {verhead_LTE }}=28 \%$ [24]. Thus the throughput of LTE is

$$
\begin{aligned}
& \text { Throughput }_{L T E} \\
& =(2.15 b p s / H z) \lambda_{b} W_{b}\left(1-O_{\text {verhead_LTE }}\right) .
\end{aligned}
$$

\section{B.2 DRMS in hybrid networks}

Under DRMS, the throughput of the hybrid networks is the sum of U-plane throughput in the small cells. According to the classical model of stochastic geometry [27], the spectral efficiency of the SC can be derived as follows:

$$
=\int_{t>0}^{S E_{\mathrm{b} \_D R M S}} \frac{1}{1+\sqrt{e^{t}-1}\left(\frac{\pi}{2}-\arctan \left(\frac{1}{\sqrt{e^{t}-1}}\right)\right)} d t,
$$

where the path loss exponent $\alpha$ is 4 and the thermal noise is ignored because the terrestrial network is an interference limited network. Thus the SE of hybrid networks is also nearly $2.15 \mathrm{bps} / \mathrm{Hz}$. Based on our previous work [20], the overhead of the U-plane, $\mathrm{O}_{\text {verhead_b }}$ is nearly $15 \%$, thus the network throughput can be obtained by

$$
\begin{aligned}
& \text { Throughput } t_{D R M S}=\text { Throughput } t_{b \_D R M S} \\
& =\int_{t>0} \frac{\lambda_{b} \mathrm{~W}_{\mathrm{b}}\left(1-\mathrm{O}_{\mathrm{verhead} \mathrm{b}}\right)}{1+\sqrt{e^{t}-1}\left(\frac{\pi}{2}-\arctan \left(\frac{1}{\sqrt{e^{t}-1}}\right)\right)} d t .
\end{aligned}
$$

\section{B.3 CRMS in hybrid networks}

Under the centralized resource management scheme, the gateway will route the traffic from the external networks to both satellite through uplink transmission and SCs through fronthaul in the terrestrial network. The SE of SC under CRMS is

$$
\begin{aligned}
& S E_{\mathrm{b}_{-} \mathrm{CRMS}}=E\left\{\log _{2}\left(1+S I N R_{b} \mid r\right) \times \mathrm{P}_{r_{-} b}(r<\eta)\right\} \\
& =E\left\{\log _{2}\left(1+\frac{P_{t b} h_{t b} r^{-\alpha}}{\sigma^{2}+\sum_{b^{\prime} \notin \Phi / b 0} \frac{P_{t b^{\prime}}{ }^{\prime} t b^{\prime}}{r^{\prime \alpha}}} \mid r\right)\right\} \times \int_{0}^{\eta} f_{r}(r) d r \\
& =\frac{1}{\ln 2} \cdot \int_{r>0} d r \int_{t>0} d t\left\{e^{\frac{-u r^{\alpha}}{P_{t b}}\left(e^{t}-1\right) \sigma^{2}} \cdot\right. \\
& \left.e^{-\pi \lambda_{\mathrm{b}} r^{2}\left(1+\left(e^{t}-1\right)^{2 / \alpha} \int_{\left(e^{t}-1\right)^{-2 / \alpha}}^{\infty} \frac{1}{1+x^{\alpha / 2}} d x\right.}\right)_{\left.2 \pi \lambda_{\mathrm{b}} r\right\} .}
\end{aligned}
$$

Neglecting the thermal noise $\sigma^{2}$ in the interference limited terrestrial networks and assuming the path loss exponent $\alpha=4$ for all the links, the throughput of SCs in the terrestrial networks can be simplified as follows :

$$
\begin{aligned}
& \text { Throughput } t_{b_{-} C R M S}=\frac{\lambda_{b}}{\ln 2} W_{b}\left(1-O_{\text {verhead_b }}\right) \\
& \int_{0}^{\infty} \int_{0}^{\pi \lambda_{b} \eta^{2}} e^{-v\left(1+\sqrt{\left(e^{t}-1\right)}\left(\frac{\pi}{2}-\arctan \left(\frac{1}{\sqrt{\left(e^{t}-1\right)}}\right)\right)\right)} d v d t .
\end{aligned}
$$

As for the SE of the LEO satellite, the probability of getting access to the satellite is also considered and the SE is derived as follows:

$$
\begin{aligned}
& S E_{s}=\mathrm{E}\left\{\log _{2}\left[1+\operatorname{SINR}_{s} \mid r\right] \times \mathrm{P}_{\mathrm{P}_{r s} s}(r>\eta)\right\} \\
& =\mathrm{E}\left\{\log _{2}\left[1+\frac{\mathrm{P}_{r s}}{k T_{\text {on_earth }} W_{s}} \mid r\right]\right\} \times \int_{\eta}^{\infty} f_{r}(r) d r \\
& =\log _{2}\left(1+\frac{P_{t s} G_{t} G_{r} \lambda^{2}}{(4 \pi d)^{2} L k T_{\text {on_earth }} W_{s}}\right) \exp \left(-\pi \lambda_{b} \eta^{2}\right) .
\end{aligned}
$$

In this paper, we consider a single satellite spot beam and transmission on the downlink, thus the interference will be dictated by the satellite antenna and can be assumed to be small compared to the thermal noise. Thus the satellite is noise rather than interference dominant. The thermal noise $\sigma^{2}=k T_{\text {on_earth }} W_{s}$ should be taken into consideration, where $k$ is the Boltzmann constant $1.3806488 \times 10^{-23} \mathrm{~J} / \mathrm{K}$ and $T_{\text {on_earth }}$ is the noise temperature of terminal. Thus the throughput of the satellite is:

$$
\begin{aligned}
& \text { Throughput }_{s} \\
& =W_{s}\left(1-O_{\text {verhead_s }}\right) \log _{2}\left(1+\frac{P_{t s} G_{t} G_{r} \lambda^{2}}{(4 \pi d)^{2} L k T_{\text {on_earth }} W_{s}}\right) \\
& \exp \left(-\pi \lambda_{b} \sqrt{\frac{P_{\mathrm{tb}}}{P_{t s}} \frac{\theta}{\lambda^{2}} \frac{(4 \pi d)^{2} L}{G_{t} G_{r}}}\right),
\end{aligned}
$$

where the path loss exponent $\alpha=4$ is used and the overhead of the satellite $O_{\text {verhead_s }}$ in the U-plane is about $15 \%$ given in our previous work [20]. The overall throughput in the U-plane in this hybrid network with $\mathrm{C} / \mathrm{U}$ split architecture is:

$$
\begin{aligned}
& \text { Throughput } t_{C R M S} \\
& =\text { Throughput }_{b_{-} C R M S}+\text { Throughput }_{s} .
\end{aligned}
$$

Obviously, the spectral efficiency under DRMS is influenced by both of the terrestrial and satellite parts simultaneously. From the derivation in (20), the asymptotic analysis can be drawn to obtain some insights on applying them to optimize the system design. With the increasing of $\lambda_{b}$ in one satellite spot beam to a relatively large value, the throughput of satellite drops to zero because $\exp \left(-\pi \lambda_{b} \sqrt{\frac{P_{\mathrm{tb}}}{P_{t s}} \frac{\theta}{\lambda^{2}} \frac{(4 \pi d)^{2} L}{G_{t} G_{r}}}\right) \rightarrow$ 0 . So the system SE under CRMS comes to $S E_{\mathrm{b}_{-} \mathrm{CRMS}} \approx$ 
$\int_{0}^{\infty} \frac{1}{1+\sqrt{e^{t}-1}\left(\frac{\pi}{2}-\arctan \left(\frac{1}{\sqrt{e^{t}-1}}\right)\right)} d t \approx 2.15 b p s / H z$, which is the lower bound of the SE in the hybrid sparse network. Typically, in the sparse network, the density of SCs is relatively low, so the satellite plays a crucial role in the hybrid network. By adjusting the access bias $\eta$ to a small value, the the SE under CRMS comes to $S E_{s} \approx \frac{\log _{2}\left(1+\frac{P_{t s} G_{t} G_{r} \lambda^{2}}{(4 \pi d)^{2} L k T_{o n} \text { earth } W_{s}}\right)}{1-\pi \lambda_{b} \eta^{2}}$, using the property that $\mathrm{e}^{\mathrm{x}}-1 \approx \mathrm{x}$ when $x \rightarrow 0$. So we can adopt these conclusions into the real network optimization. If the traffic requirement is high in sparse network, more SCs have to be deployed to achieve higher throughput, with the spectral efficiency approaching to constant value $S E_{\mathrm{b}_{-} \mathrm{CRMS}} \approx 2.15 \mathrm{bps} / \mathrm{Hz}$. On the contrary, little SCs will be deployed in low traffic scenario, the access bias $\eta$ can be adjusted to increase the SE, which are verified in Section VI.

\section{Power Consumption}

The overall energy consumption consists of two parts: the power consumption of the SCs and the satellite gateway (P-GW and S-GW). Although the satellite is operated by solar panel$\mathrm{s}$ and batteries which are limited in energy capacity, the power of the satellite is not taken into consideration due to the fact that the solar energy is renewable, sustainable and not included in the grid power consumption, so that the one-off energy to launch the satellite will be very small compared with the power consumed over the useful life. Furthermore, the power consumed in the terminal side can be ignored, because the access network (base stations) already accounts for nearly $80 \%$ of the overall power consumption [31]. Also, the power used for calculating and transmitting in terminals are even smaller, compared with the power cost by the brightness of the screen and apps updating in the background.

The power model of the SCs is modelled as follows according to the reference [32]:

$$
P_{b}=\alpha^{\prime} P_{t b}+P_{b 0},
$$

where $P_{t b}$ is the transmission power related to the traffic load, $\alpha^{\prime}$ is the increase power coefficient and $P_{b 0}$ is the static power of SC.

The gateway in the hybrid network plays an important role in the following areas:

- Calculating and storing details about the subscribers and the related mobility management information

- Sending back the processed information to the satellite in the uplink

- Routing the traffic to the external networks

So the energy consumption of the gateway is calculated as the sum of the energy cost in three areas:

$$
P_{\text {gateway }}=P_{g t x}+P_{c}+P_{g b h}
$$

where $P_{g t x}$ is the uplink traffic transmission power from the gateway to the satellite. Taking advantage of the link budget equation, this part of the power consumption is given as:

$$
P_{g t x}=\frac{\left(2^{\text {Throughput }} / W_{\mathrm{g}}-1\right) \times \mathrm{kT}_{\text {on_satellite }} W_{\mathrm{g}}}{\frac{G_{t}^{\prime} G_{r}^{\prime} \lambda^{\prime 2}}{(4 \pi d)^{2} L^{\prime}}},
$$

where $W_{\mathrm{g}}$ is the bandwidth of gateway, $T_{\text {on_satellite }}$ and $\lambda^{\prime}$ are the noise temperature and uplink wavelength of the satellite, and $G_{t}{ }^{\prime}, G_{r}{ }^{\prime}, L^{\prime}$ are the transmitter and receiver antenna gains, uplink atmosphere loss respectively. The static computing power consumption $P_{c}$ is given in Table 3 in [33], and $P_{g b h}$ is the energy consumption of fronthaul back to the external networks [34]:

$$
P_{g b h}=\frac{\text { Throughput }_{b}+S E_{s} \times W_{s}}{100 M b p s} \times 50 W,
$$

where Throughput $_{b}$ is the result of Throughput th_DRMS $_{-}$and Throughput b_CRMS $_{-}$in (15) and (17) respectively.

\section{Energy Efficiency}

The energy efficiency of the networks is modeled as the throughput of the U-plane per watt consumed in the power grid. So the EE of LTE networks, DRMS and CRMS of hybrid C/U split networks can be expressed as follows:

- EE of LTE networks

$$
E E_{\text {Sparse_LTE }}=\frac{\text { Throughput }_{L T E}}{\lambda_{b} P_{b}+P_{c}+P_{g b h_{-} d}},
$$

- EE of hybrid networks with DRMS

$$
E E_{\text {Sparse_DRMS }}=\frac{\text { Throughput }}{\lambda_{b} P_{b}+P_{c}+P_{g b h \_d}},
$$

- EE of hybrid networks with CRMS

$$
E E_{\text {Sparse_CRMS }}=\frac{\text { Throughput }}{\lambda_{C R M S}}{ }_{\lambda_{b}+P_{c}+P_{g b h \_c}+P_{g t x}} .
$$

\section{Ultra-dense Networks Analysis}

In the ultra-dense networks, there are numerous small cells in the terrestrial network, where some of the base stations might have no users to serve. The closed access policy is applied so that the SUE and delay-sensitive PUE get access to the small cells for delay-tolerant PUEs supported by the satellite. In this section, the service probability, throughput, power consumption and energy efficiency are analysed and different resource management schemes, bandwidth allocation methods, and on-off approaches are considered.

\section{A. Service Coverage}

In the ultra-dense scenario, the small cells can support higher data-rates and good coverage, so that the QoS of users will be of greater significance, including the rate and delay constraints. Thus the definition of service coverage probability is introduced.

Definition 2 (Service Coverage) The service coverage probability is the probability that a randomly chosen user can achieve a targeted data rate threshold $U$ :

$$
S_{\mathrm{cov}}(U) \triangleq P(R>U)
$$

which is equivalent to the average fraction of UEs in the networks with the achieved data rate $R$ larger than the threshold $U$.

Assuming $\mathrm{X}$ is a random variable of the size of a typical Voronoi cell, using the ergodicity of the PPP, the probability density 
function of the area distribution of a typical small cell is given in the following lemma [35].

$$
f_{X}(x)=\frac{3.5^{3.5}}{\Gamma(3.5)} x^{2.5} e^{-3.5 x} .
$$

Lemma 1: let the random variable $N_{b}$ denote the total numbers of the users in one Voronoi cell to which a randomly chosen user belongs. Thus the PDF of $N_{b}$ is given as follows:

$$
\begin{aligned}
& P\left(N_{b}=n\right) \\
& =\sum_{n \geq 1} \int_{0}^{\infty} P\left(N_{b}^{\prime}=n-1 \mid X=x\right) f_{X}(x) d x \\
& =\int_{0}^{\infty} \exp \left(-\frac{\lambda_{b u} x}{\lambda_{b}^{\prime}}\right) \frac{\left(\frac{\lambda_{b u} x}{\lambda_{b}^{\prime}}\right)}{(n-1) !} f_{X}(x) d x \\
& =\frac{3.5^{4.5}\left(\lambda_{b u} / \lambda_{b}^{\prime}\right)^{(n-1)} \Gamma(n+3.5)}{\Gamma(4.5)(n-1) !\left(\lambda_{b u} / \lambda_{b}^{\prime}+3.5\right)^{(n+3.5)}},
\end{aligned}
$$

where $\Gamma(x)=\int_{0}^{\infty} \exp (-t) t^{x-1} d t$ is the gamma function, $\lambda_{b}^{\prime}$ and $\lambda_{b u}$ are the density of SC and users per $\mathrm{km}^{2}$.

In addition, resulting from [36] the mean load of one base station can be simplified as follows:

$$
\begin{aligned}
& E\left[N_{b}\right]=1+\frac{\lambda_{b u}}{\lambda_{b}^{\prime}} E\left[C^{2}(1)\right] \\
& =1+1.28 \frac{\lambda_{b u}}{\lambda_{b}^{\prime}}
\end{aligned}
$$

where $E\left[C^{2}(1)\right]=1.28$ comes from [37].

Theorem 1 (SC Service Coverage) For a typical user to get access to a $\mathrm{SC}$, the service coverage probability is

$$
\begin{aligned}
& S_{\mathrm{cov}}(U)=P(R>U) \\
& =E_{N}\left[P\left(\frac{w_{b}}{N} \log _{2}(1+S I N R)>U\right)\right] \\
& \stackrel{(b)}{=} E_{N}\left[P_{\mathrm{cov}}\left(2^{\frac{U N}{w_{b}}}-1\right)\right] \\
& \stackrel{(c)}{=} \sum_{n \geq 1} p(N=n) P_{\mathrm{cov}}\left(2^{\frac{n U}{w_{b}}}-1\right) \\
& =\sum_{n \geq 1} \frac{3.5^{4.5}\left(\lambda_{b u} / \lambda_{b}^{\prime}\right)^{(n-1)} \Gamma(n+3.5)}{\Gamma(4.5)(n-1) !\left(\lambda_{b u} / \lambda_{b}^{\prime}+3.5\right)^{(n+3.5)}} \pi \lambda_{b}^{\prime} \\
& \cdot \int_{0}^{\infty} \mathrm{e}^{-\pi \lambda_{b}^{\prime} v\left(1+\rho\left(2^{\frac{n U}{w_{b}}}-1, \alpha\right)\right)} d v, \\
& \rho(T, \alpha)=T^{2 / \alpha} \int_{T^{-2 / \alpha}}^{\infty} \frac{1}{1+x^{\alpha / 2}} d x,
\end{aligned}
$$

where (b) is from the definition of SINR coverage in (9) and (c) is utilized from [36] [38]. $\alpha$ is the path loss exponential factor in terrestrial networks. The available bandwidth of the smal1 cell $w_{b}$, with overhead cost taking into account, varies under different bandwidth allocation schemes. In this ultra-dense network, $U$ is the QoS rate constraints, used as $U_{P U E}$ and $U_{S U E}$ respectively for PUE and SUE.

Corollary 1 (Mean SC Service Coverage) For a specific case, based on the approximation of $E_{N}[S(N)] \approx S(E[N])$ in [36], when all of the small cells are of equal load with the mean value and path loss exponent 4 and with noise neglected, we have the following property:

$$
\begin{aligned}
& \bar{S}_{\mathrm{cov}}(U)=P_{\mathrm{cov}}\left(2^{\frac{E[N] U}{w_{b}}}-1\right) \\
& =1 /(1+v(\pi / 2-\arctan (1 / v))),
\end{aligned}
$$

$$
v=\sqrt{2^{\left(1+1.28 \frac{\lambda_{b u}}{\lambda_{b}^{\prime}}\right) \frac{U}{w_{b}}}-1 .}
$$

Theorem 2 (Mean Satellite Service Coverage) For the users to get access to the satellite in the spot beam with the area of $A_{s}$, utilizing the LMS channel property in (2), the mean satellite service coverage is

$$
\begin{aligned}
& \bar{S}_{\operatorname{cov} 2}(U)= \\
& 1-A_{0}\left(\frac{2^{\frac{U \lambda_{s u} A_{s}}{w_{s}}}-1}{\bar{\gamma}_{s d}}\right){ }_{1} F_{1}\left(m, 2, B_{0} \frac{2^{\frac{U \lambda_{s u} A_{s}}{w_{s}}}-1}{\gamma_{s d}}\right) \\
& -\frac{A_{0} \Omega}{4 b_{0}}\left(\frac{2^{\frac{U \lambda_{s u} A_{s}}{w_{s}}}-1}{\bar{\gamma}_{s d}}\right){ }_{2}^{2} F_{2}\left(2, m ; 3,1 ; B_{0} \frac{2^{\frac{U \lambda_{s u} A_{s}}{w_{s}}}-1}{\bar{\gamma}_{s d}}\right),
\end{aligned}
$$

where $\lambda_{\text {su }}$ is the number of users served by the satellite in Uplane per $\mathrm{km}^{2}$, and $w_{s}$ is the available bandwidth of the satellite taking consideration of the overhead cost. The related channel parameters are defined in (4) - (6).

Proof: See Appendix VII.

It should be noted that, in ultra-dense networks, the LMS channel is used instead of the simple path loss model adopted for sparse networks, because the service coverage is needed and the QoS constraints become an important metric.

\section{B. Service Throughput}

In the ultra-dense networks, the users have a higher probability of having an improved SINR from the attached base station. Although the SINR coverage is satisfied, the base station may not provide the data rate threshold required from the terminal. Thus the service throughput is defined as follows.

Definition 3 (UE Service Throughput) Achievable throughput per user in ultra-dense networks:

$$
T(U) \triangleq E[R \mid R>U]
$$

where $R$ is the instant data rate of a typical user in a ultra-dense network, and $U$ is the threshold of the rate QoS threshold for this type of terminal.

Theorem 3 (Mean UE Achievable Throughput) Utilizing the definition of SINR coverage function in (9), the achievable throughput of user is

$$
\bar{T}(U)=U+\frac{1}{\theta \ln 2} \int_{2^{\theta U}-1}^{\infty} \frac{P_{\mathrm{cov}}(\mathrm{y})}{P_{\mathrm{cov}}\left(2^{\theta U}-1\right)(1+y)} \mathrm{d} y .
$$

$\theta=E[N] / w$ is the average number of users per available bandwidth unit, where $E[N]$ is the total active users be scheduled by the networks and $w$ is the available bandwidth, used as $w_{b}$ and $w_{s}$ for the SC and satellite respectively.

In specific, the achievable throughput of users get access to a terrestrial network is

$$
\begin{aligned}
& \bar{T}_{b}(U)=U+\frac{1}{\theta \ln 2} \\
& \int_{2^{\theta U-1}}^{\infty} \frac{1+\sqrt{\left(2^{\theta U}-1\right)}\left(\pi / 2-\arctan \left(1 / \sqrt{\left(2^{\theta U}-1\right)}\right)\right)}{1+\sqrt{y}(\pi / 2-\arctan (1 / \sqrt{y}))} \frac{1}{1+y} d y
\end{aligned}
$$

and the achievable throughput of satellite users is shown as

$$
\bar{T}_{s}(U)=U+\frac{1}{\theta \ln 2} \int_{2^{\theta U}-1}^{\infty} \frac{1-F_{r s d}(y)}{1-F_{r s d}\left(2^{\theta U}-1\right)} \frac{1}{1+y} d y
$$


Table 2. Simulation Parameters.

\begin{tabular}{|c|c|c|c|c|}
\hline & Parameter & Value & Parameter & Value \\
\hline \multirow{5}{*}{ Satellite } & $P_{t s} G_{t}(E I R P)$ & $54.4 d B W$ & $W_{s}$ & $30 M H z$ \\
\hline & Overhead_s & $15 \%$ & $G_{r}$ & $0 d B$ \\
\hline & $\lambda_{b}$ (Sparse Networks) & $1 \sim 40$ & $\lambda_{b}$ (Ultra-dense Networks) & $1000 \sim 5000$ \\
\hline & $\lambda$ & $137 \mathrm{~mm}$ & $L$ & $0 d B$ \\
\hline & $d$ & $1000 \mathrm{~km}$ & $T_{\text {on_satellite }}$ & $26 d B K$ \\
\hline \multirow{5}{*}{ Small Cell } & $P_{t b}$ & $0 \sim 4 W$ & $P_{b 0}$ & $28.7 \mathrm{~W}$ \\
\hline & $\alpha^{\prime}$ & 16 & $W_{b}$ & $10 M H z$ \\
\hline & $T_{\text {on_earth }}$ & $290 K$ & $u$ & 1 \\
\hline & $T$ & $0 d B$ & $O_{\text {verhead_b }}$ & $15 \%$ \\
\hline & $\theta$ & $-125 d B,-145 d B,-165 d B$ & & \\
\hline \multirow{3}{*}{$\begin{array}{c}\text { Gateway } \\
-\end{array}$} & $\lambda^{\prime}$ & $50 \mathrm{~mm}$ & $W_{g}$ & $10 M H z$ \\
\hline & $G_{t}^{\prime}$ & $40 d B$ & $G_{r}^{\prime}$ & $16 d B$ \\
\hline & $P_{c}$ & $355 W$ & $L^{\prime}$ & $0 d B$ \\
\hline
\end{tabular}

where the QoS threshold $U$ is the rate threshold of PUE and SUE, representing as $U_{P U E}$ and $U_{S U E}$ respectively. The function of $F_{r s d}(\gamma)$ is defined in [30], where $\gamma_{s d}$ is given in (4).

Proof: See the Appendix D in [39].

Corollary 2 (Mean SC Achievable Throughput) Based on the results in Theorem 3 and Lemma 1, the mean throughput of one typical small cell in hybrid networks is shown as below

$$
\bar{T}_{h \_b}=E\left[N_{b}\right] \bar{S}_{\mathrm{cov}}(U) \bar{T}_{b}(U),
$$

where $E\left[N_{b}\right]$ is the number of users scheduled by one typical small cell.

Corollary 3 (Network Throughput) In addition, we can calculate the throughput of the system by calculating each users' achievable data rate and summing them to get the total throughput of the networks. The definition of the throughput of the network is:

$$
\text { Throughput }=\lambda_{u} A_{s} S_{\text {cov }}(U) T(U),
$$

where $\lambda_{u} A_{s}$ is the total number of users could achieve the targeted rate threshold in the networks. The average throughput of terrestrial and satellite networks are given respectively:

$$
\begin{aligned}
& \bar{T}_{h b \_a l l}=\lambda_{b u} A_{s} \bar{S}_{\text {cov }}(U) \bar{T}_{b}(U), \\
& \bar{T}_{h s}=\lambda_{s u} A_{s} \bar{S}_{\operatorname{cov} 2}(U) \bar{T}_{s}(U) .
\end{aligned}
$$

\section{Energy Efficiency}

Utilizing the results in (21-24), the EE of the hybrid networks can be expressed as:

$$
E E_{h}=\frac{\bar{T}_{h b a l l}+\bar{T}_{h s}}{\lambda_{b}^{\prime} A_{s} P_{b}+P_{c}+P_{g b h}+P_{g t x}} .
$$

\section{On-off Strategy}

In this subsection, the sleeping of SCs is considered under two modes, RSM and TBM, introduced in Section III. Under the random sleep mode, the small cells are configured with equal sleep probability $\varsigma$, which is equivalent to the situation discussed above with the density of SC $\varsigma \lambda_{b}^{\prime}$. Under the traffic based mode, using the results of [40], the small cells can sleep with a probability of

$$
\varsigma=\left(1+3.5^{-1} \lambda_{b u} / \lambda_{b}^{\prime}\right)^{-3.5},
$$

where there are no active users in the coverage of one typical $\mathrm{SC}$. The service coverage probability is given as:

$$
\begin{aligned}
& \bar{S}_{\text {cov_} \_T B M}(U)=1 /(1+(1-\varsigma) v(\pi / 2-\arctan (1 / v))), \\
& v=\sqrt{2^{\left(1+1.28 \frac{\lambda_{b u}}{\lambda_{b}^{\prime}}\right) \frac{U}{w_{\mathrm{b}}}}-1,}
\end{aligned}
$$

where $v$ is the same as that in (35) but the service coverage probability is affected by $\varsigma$. It should be noted, that the TBM on-off strategy has no influence on the density of small cells in the access procedure, so that the users should select the best small cell from all of the SCs to gain access with no effect on the nearest distance distribution $r$ in (1). After this procedure, the base stations with no users can be put into sleep mode, and the interference conditions changed. With the increase of SC density $\lambda_{b}^{\prime} \rightarrow \infty$, the probability of sleeping is increasing with higher $\varsigma \rightarrow 0$ and $v \rightarrow \sqrt{2^{\frac{U}{w_{\mathrm{b}}}}-1}$, and the whole service coverage $\bar{S}_{\text {cov_TBM }}(U) \rightarrow 1$. Thus it is clear that the service coverage probability increases with density of SCs. In addition, larger QoS request $U$ leads to larger $v$, which degrading the network coverage performance. In section VI, numerical simulation results match these asymptotic analysis.

\section{Performance Evaluation and Main Application}

In the following, we use the default values in Table 2 to illustrate the main results, where the key parameters in the terrestrial networks are based on the results of the EARTH Project and the satellite parameters are obtained from [41]. In the terrestrial network, small cells are deployed at $3.5 \mathrm{GHz}$, whilst the satellite downlink channel operates in S-band (2-3 GHz). For the uplink channel, from the gateway to the satellite, C-band $(6 \mathrm{GHz})$ is assumed and a $2 \mathrm{~m}$ antenna is used at the gateway.

\section{A. Performance in Sparse Networks}

Fig. 2 (a) illustrates the U-plane SINR coverage probability in the hybrid satellite terrestrial networks. Comparisons are made between two resource management schemes: CRMS and DRMS. For DRMS strategy, it is shown that the U-plane coverage probability remains the same under certain outage threshold $T$ for the hybrid networks under the DRMS strategy, which does not affected by the density $\lambda_{b}$ or the transmission power $P_{t b}$ of $\mathrm{SC}$. This is because the satellite only provides $\mathrm{C}$-plane coverage 
(a)

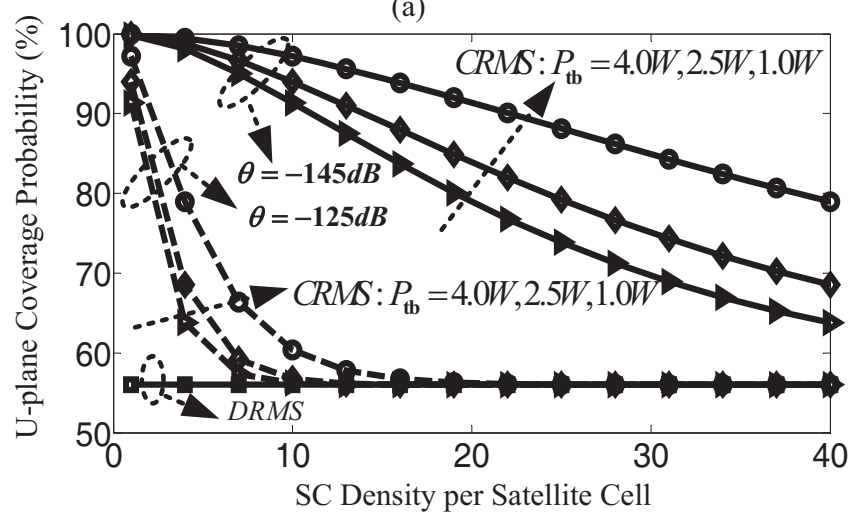

(b)

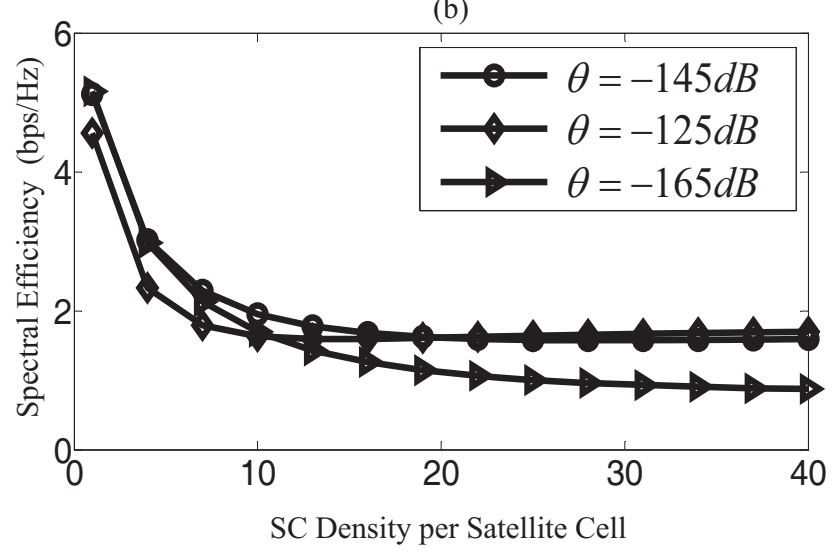

Fig. 2. U-plane coverage in sparse networks: (a) U-plane coverage probability under DRMS and CRMS with different SC density per satellite cell $\lambda_{b}$, various transmission power of SC $P_{t b}$ and access bias $\theta$; (b) Spectral efficiency under various bias with CRMS.

(a)

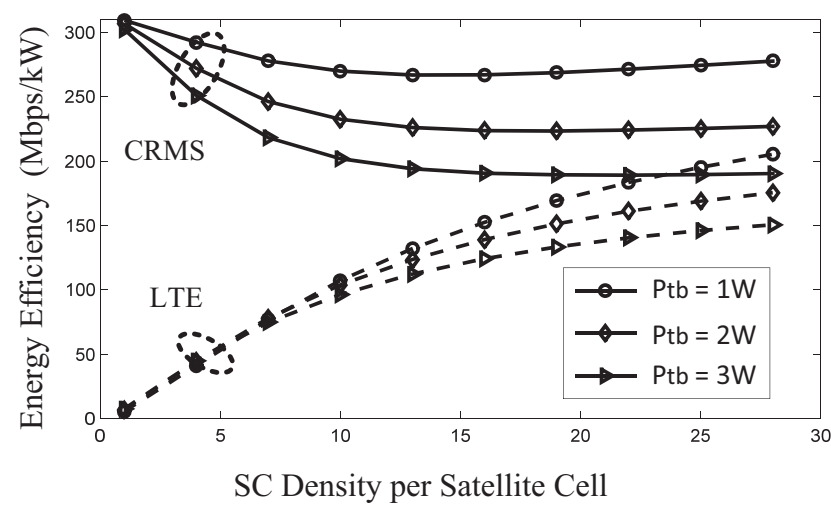

(b)

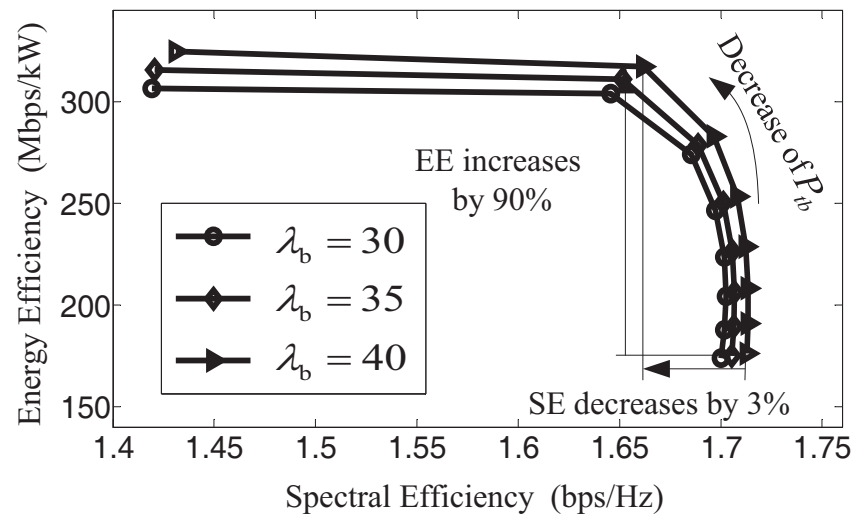

Fig. 3. SE and EE results in sparse networks, where $P_{t b}$ is the transmission power per SC and $\lambda_{b}$ is the SC density per satellite cell: (a) Network EE comparison between the hybrid network with CRMS and the LTE network; (b) Tradeoff between SE and EE in hybrid networks with CRMS.

and RRC-connection mobility control information under DRMS. So these two factors can not affect the SINR in the network. Furthermore, it is seen that the U-plane coverage performance under CRMS is much better than DRMS, as the satellite can achieve large SINR without the interference from SCs. In addition, the larger bias factor $\theta, \mathrm{SC}$ density $\lambda_{b}$ and $\mathrm{SC}$ transmission power $P_{t b}$ affect the probability to gain access to the satellite, resulting in lower coverage probability. The hybrid networks with CRMS can achieve around $57 \%$ coverage probability gain in sparse networks (e.g., $\lambda_{b}=5$ ) than for the DRMS network.

The spectral efficiency performance of the hybrid network with CRMS is illustrated in Fig. 2 (b). For comparison, the SE of DRMS is set as the baseline, which has been verified theoretically as nearly $2.15 \mathrm{bps} / \mathrm{Hz}$ in Section IV. It can be found that the SE of the hybrid network with CRMS is obviously larger than SE with DRMS when the density of SCs is small. In addition, the bias $\theta$ significantly influences the probability for the UE to gain access to the satellite or SC, and the SC density $\lambda_{b}$ has quite a different impact on the SE of the networks. With small bias, e.g., $\theta=-165 d B$, the UE can hardly gain any access to the SCs, so that the performance degrades quickly with the increase of $\lambda_{b}$. As a consequence, the advantages of the satellite weakens with large bias, e.g., $\theta=-125 d B$. In this way, a proper bias with a median value of $\theta=-145 \mathrm{~dB}$, should be used to gain benefit from both the SC and the satellite. The constant path loss factor is reflected by the bias, so that the absolute value is small. Note that in terrestrial macro cells the bias $\theta$ is around $0 d B$, but here due to the lower received power from the satellite we have a much smaller value. Though the received power is low in the satellite network, the SINR could also be larger than that in the terrestrial network, because of the severe interference between small cells. Combining the results shown in Fig. 2 (a) and (b), it can be concluded that by adjusting the bias factor, the network with CRMS can achieve much better U-plane SINR coverage probability and spectral efficiency by making the most use of the satellite in the sparse network scenario. So in the following, we mainly study the performance of the hybrid network with CRMS in the sparse network, and make comparisons with LTE networks.

With the proper bias $\theta=-145 d B$, Fig. 3 (a) shows the EE comparison between hybrid networks under CRMS strategy and LTE networks. It is seen that initially the EE grows with the increase of SC density in the LTE network, resulting from the tradeoff between higher throughput and the static power consumption of the gateway and SC. It then stabilizes as both the power consumption and throughput vary linearly with the den- 

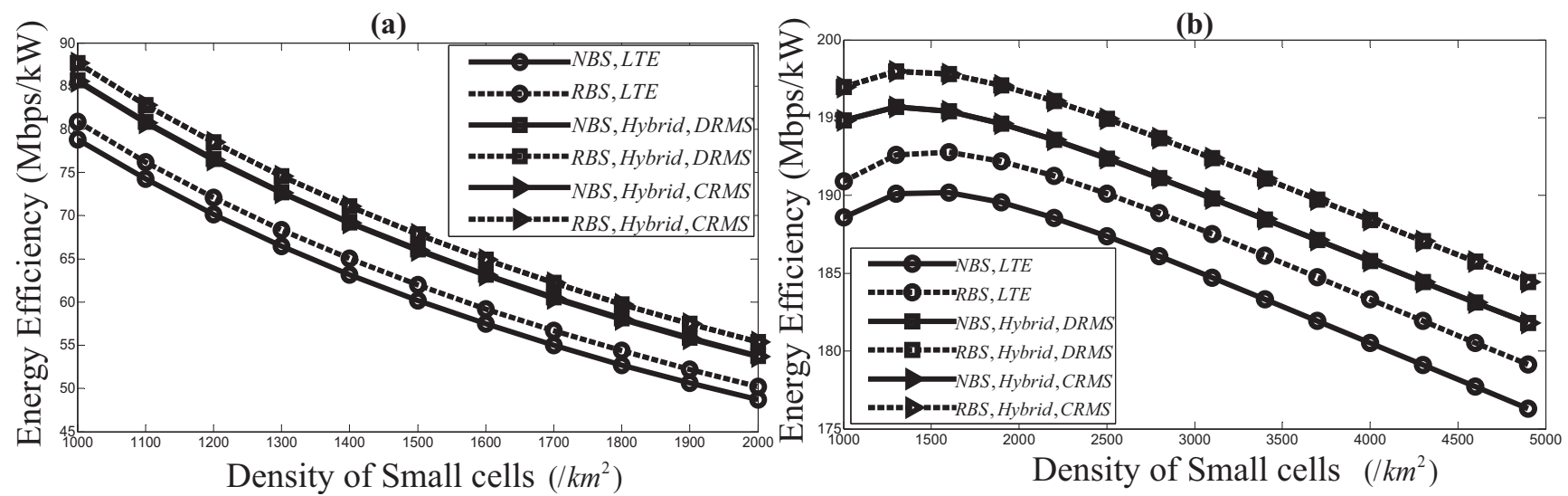

Fig. 4. Energy efficiency of the network under various strategies : (a) Typical load requirement with 500 active SUEs per $\mathrm{km}^{2}$; (b) Ultra-high load requirement with 25000 active SUEs per $\mathrm{km}^{2}$.

sity of the SCs. For the hybrid networks with CRMS, the results are totally different. The EE reaches quite a high value when the density of small cells is low, benefiting from the higher probability to gain access to the satellite and higher throughput from the satellite. With the increase in density of the SCs, the EE shows a downward trend and finally remains constant with that under the LTE network. In addition, the probability to gain access to the satellite and the power consumption are affected by the transmission power of the SCs $P_{t b}$, so that the EE in both LTE and CRMS networks will decrease with the growth of $P_{t b}$. It is seen that the networks with CRMS strategy achieve higher network EE than in LTE systems, especially for sparse networks (e.g., $\lambda_{b}=25$ ) where the EE gain is nearly $40 \%$.

In sparse networks, shown in Fig. 3 (b), larger $P_{t b}$ helps to increase SE and EE initially, but then EE drops quickly and SE remains stable with the increase of $P_{t b}$. In the sparse networks (e.g., $\lambda_{b}=40$ ), compared with the SE optimized parameter setting, realistic transmission power $P_{t}$ (e.g., $0.5 W$ ) helps to increase EE by approximately $90 \%$ with only $3 \%$ SE loss. By utilizing this tradeoff, the network energy consumption can be greatly saved under certain constraints of SE performance.

Main conclusions for sparse networks can be summarized as follows:

- In hybrid networks, by making full use of satellite, the CRM$\mathrm{S}$ can achieve higher SE, and the coverage probability can be increased by around $57 \%$ over the DRMS strategy;

- By choosing a proper bias factor, the SE in the hybrid network with CRMS can benefit from both satellite and terrestrial network;

- The hybrid network with CRMS can achieve nearly $40 \%$ EE gain over that of the LTE network;

- There exists obvious trade-offs between EE and SE in the hybrid network with CRMS strategy, where proper choice of transmission power helps to increase network EE by approximately $90 \%$ with only $3 \%$ SE loss.

In conclusion, the hybrid network shows better SE and EE performance than the LTE network. By utilizing the appropriate value of the SC transmission power and bias factor, the centralized resource management scheme is more suitable for the sparse network than the distributed resource management scheme.

\section{B. Performance in Ultra-dense Networks}

Different from the sparse network, which is mainly concentrated in the overall coverage and system performance. In this subsection, in ultra-dense networks, energy efficiency is discussed under different bandwidth allocation schemes (NBS and RBS) and different resource management schemes (DRMS and CRMS). Two types of on-off approaches are compared under different QoS and load, and the service coverage probabilities under these approaches are illustrated in the end.

Fundamental detailed environment parameters are given as follows. The spot beam coverage of the LEO satellite is assumed as $R=200 \mathrm{~km}$ [41], and the coverage area is $A_{s}=\pi R^{2}$. The density of small cells varies from $1000 \sim 5000$ per $\mathrm{km}^{2}$ with radius even less than 10 meters [4]. Note that this assumes an extreme condition in which small cells fill the satellite beam. In practice, the situation will be reduced as dense cells will only occupy a percentage of the satellite coverage. The typical QoS requests $U$, for PUE and SUE, represented by the rate threshold, are assumed as $1.6 \mathrm{kbps}$ (200 bytes) and $160 \mathrm{kbps}$ respectively. According to [42] [43], we assume the active rate of PUE and SUE as $2 \%$ and $25 \%$, and the active number of PUEs and SUEs are 460 and 500 per $\mathrm{km}^{2}$ in a typical load mode respectively. In the extreme situation with ultra-high load, the density of active SUEs is assumed to reach 25000 per $\mathrm{km}^{2}$ to simulate the large traffic density in future networks.

In Fig. 4, the energy efficiency of the network is illustrated under various strategies. In Fig. 4 (a) with a typical traffic load, 500 active SUEs per $\mathrm{km}^{2}$, the increase of throughput is slower than the small cell static power increase. The energy efficiency shows a downward trend with the increase in SCs. In addition, the energy efficiency of the hybrid system is nearly $5 \%$ higher than that of the LTE networks, benefiting from the C-plane coverage by the satellite. The RBS bandwidth allocation scheme is also better than the NBS scheme because the greater bandwidth allocated to SUEs achieves higher throughput. However, the offloading of delay-tolerant PUEs to the satellite, in the CRMS strategy, is no longer appropriate for ultra-dense networks, which shows no advantage over the DRMS strategy. This is quite different from that in sparse networks, due to the fact that the advantage of satellite bandwidth is very limited compared with the 
(a)

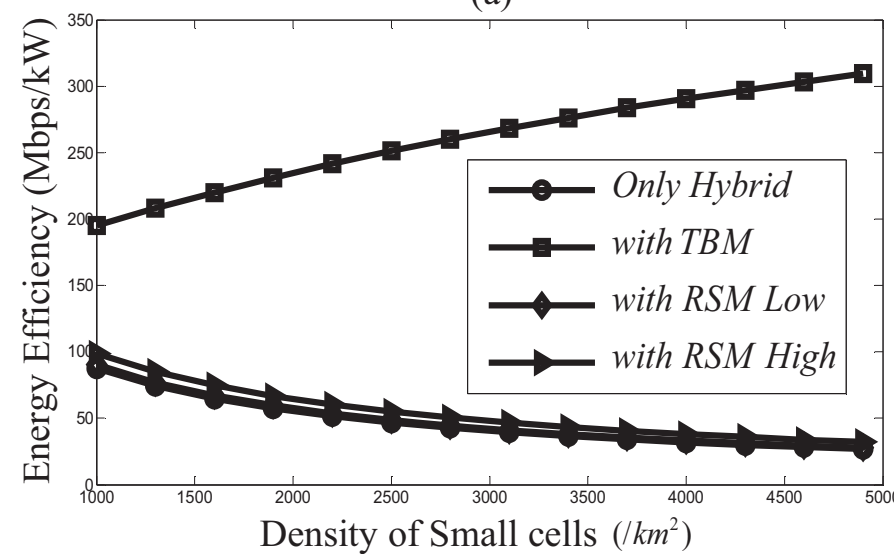

(c)

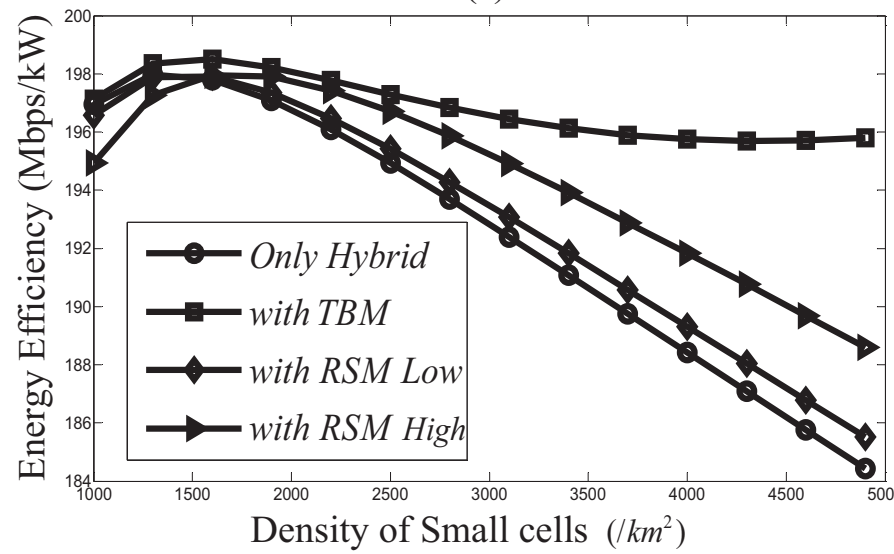

(b)

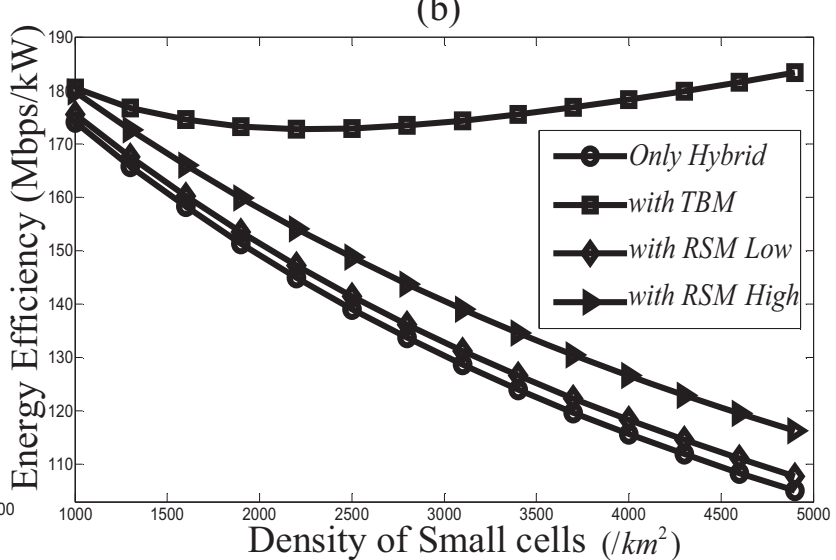

(d)

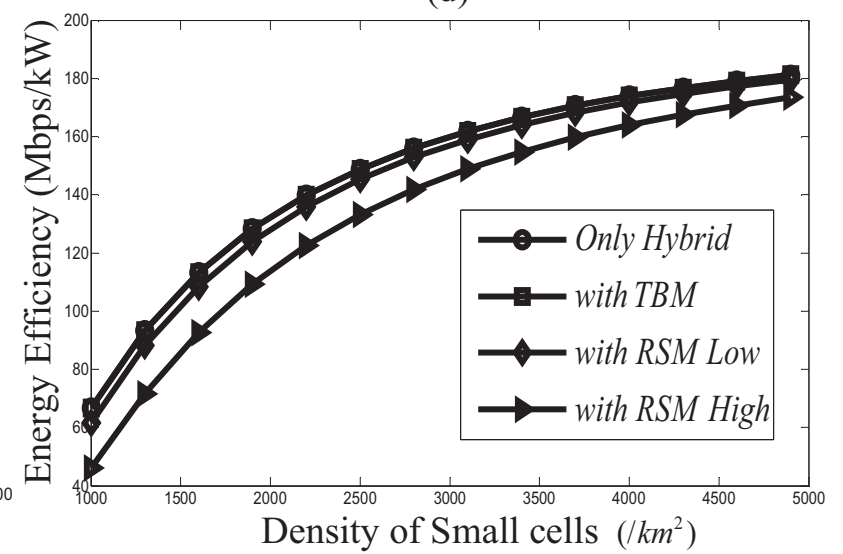

Fig. 5. Energy efficiency comparison with different QoS under various on-off strategies, and the RSM strategies is configured with higher (15\%) and lower sleep proabbility (5\%): (a) SUEs with typical load and low QoS requirement (500 active SUEs per $\mathrm{km}^{2}, 160 \mathrm{kbps}$ ); (b) SUEs with typical load and high QoS requirement (500 active SUEs per $\mathrm{km}^{2}, 250 \mathrm{kbps}$ ); (c) SUEs with ultra-high load and low QoS requirement (25000 active SUEs per $\mathrm{km}^{2}$, $160 \mathrm{kbps}$ ); (d) SUEs with ultra-high load and high QoS requirement (25000 active SUEs per $\mathrm{km}^{2}, 250 \mathrm{kbps}$ ).

huge number of SCs and the power consumption of the gateway. Also, the fronthaul consumption under CRMS is much higher when more information needs to be transmitted via the satellite.

In Fig. 4 (b) with ultra-high the traffic load, 25000 active SUEs per $\mathrm{km}^{2}$. The energy efficiency increases towards the peak value and then reduces gradually with the increase of small cells. Under this scenario, the appearance of a peak value results from the tradeoff of throughput and energy consumption. The EE of the hybrid network is nearly $3 \%$ higher than in LTE networks. The reason why the benefit is not as large as in sparse networks is that the satellite bandwidth is small compared with the overall bandwidth of the SCs.

In LTE networks, as the coverage needs to be maintained, small cells can not easily get into sleep mode. However, under the hybrid architecture, the satellite can provide wide coverage and help the users to achieve C-plane connection until they get access to the proper base station. So the sleep mode can help reduce power consumption and improve the EE of the network as shown in Fig. 5.

In Fig. 5 (a) with a typical load, it can be found that the hybrid network with RSM mode shows slightly higher EE than the hybrid network. As a contrast, the energy efficiency under TBM mode is much higher than the RSM strategies. It can be inter- preted as that the TBM strategy can provide higher probabilities for users to gain access to the best small cell and also reduce interference and power consumption for the networks. The higher the number of SCs, the more choices there are for terminals to gain access, so the expected distance is smaller resulting in higher EE. With higher QoS requirement, shown in Fig. 5 (b), the energy efficiency with TBM reduces at first because more new $\mathrm{SCs}$ are chosen resulting in more energy consumption. Then the EE with TBM increases, benefiting from higher throughput. Compared with the LTE network, the energy efficiency of the hybrid network with TBM strategy can be improved by nearly $500 \%$ and $80 \%$ respectively under low and high QoS requirement with typical load.

Fig. 5 (c) shows the ultra-high traffic condition and low QoS requirement. The TBM strategy can help to increase the networks EE by $6.5 \%$ and $12 \%$ compared with the hybrid system without "on-off" strategy and the LTE system respectively. In contrast, the energy efficiency of the networks with ultra-high traffic density and high QoS requirement is shown in Fig. 5 (d), more small cells provide higher throughput and a lower probability for small cells to go to sleep mode, resulting in the same EE between hybrid networks with and without TBM strategy.

The service coverage probability is analysed under various 
(a)

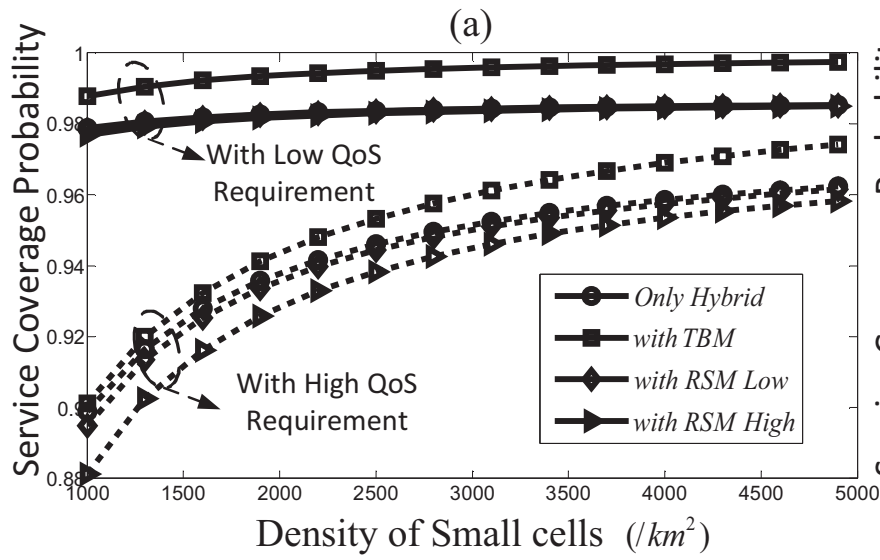

(b)

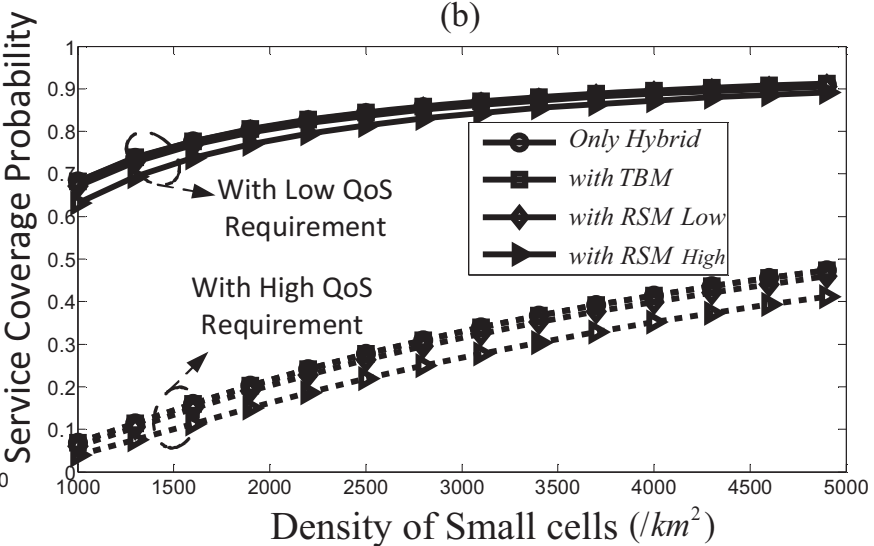

Fig. 6. Service coverage probability in ultra-dense network with different bandwidth allocation schemes: (a) Typical load requirement with 500 active SUEs per $\mathrm{km}^{2}$; (b) Ultra-high load requirement with 25000 active SUEs per $\mathrm{km}^{2}$.

on-off schemes, and the results are shown in Fig. 6. As analysed in Section V, the increasing of SC density improves the network service coverage and higher QoS requirements make the bandwidth competition more fierce and the coverage probability is much lower than that with lower QoS requirements.

In addition, with RSM strategy, higher sleep probability of SCs leads to lower service coverage probability. Under typical traffic load in Fig. 6 (a), the TBM strategy has higher coverage performance than the hybrid system, as it reduces the number of SCs and helps to decrease the interference. However, under high traffic load Fig. 6 (b), these benefits become quite small as all small cells have been put into use, so that the TBM achieves the same coverage performance as the LTE system. All these conclusions match the asymptotic results.

Main conclusions in ultra-dense networks, including the resource management schemes, bandwidth allocation methods, on-off approaches, can be summarized as follows:

- In ultra-dense networks, the role of the satellite is to provide $\mathrm{C}$-plane coverage, rather than providing data service to the terminals. The CRMS strategy has no advantages over the DRMS strategy in terms of the energy efficiency;

- The RBS scheme is better than the NBS scheme in the ultradense network, because larger throughput can be achieved to the SUEs to improve the overall network throughput;

- The EE can be enhanced dramatically by utilizing the on-off strategy of the C/U split architecture. Even under high QoS requirement constraints, the energy efficiency can be improved by over $80 \%$ over the LTE networks in typical traffic load;

- In terms of the on-off strategy, the TBM strategy can achieve higher energy efficiency and better service coverage probability than the RSM strategy.

\section{Main Applications}

The results have illustrated that the satellite can be used appropriately in future $5 \mathrm{G}$ wireless networks, to improve data throughput, coverage and energy efficiency.

In sparse networks, the satellite is able to maintain coverage for users, especially in remote places. In addition, the advantages of the satellite bandwidth can be used to improve the energy efficiency. Thus the tradeoff between SE and EE in sparse networks can be utilized to increase EE significantly with very slight SE decrease.

However, in ultra-dense networks, the user requirements are of higher importance, where the metric of SINR coverage probability is substituted by the service coverage probability. In this scenario, the human type communication SUE is of higher priority, thus the requirement-based bandwidth allocation scheme is much better than the number-based strategy. In addition, the satellite hardly enhances the throughput in ultra-dense networks, the CRMS strategy, to offload some delay-tolerant PUE to the satellite, is not appropriate. It is worth noting that the role of the satellite in the ultra-dense scenario is to increase the probability for small cells to get into sleep. Utilizing the traffic-based mode strategy, higher energy efficiency and service coverage probability can be got.

\section{Conclusions and Future Work}

In this paper, an end-to-end hybrid satellite terrestrial network was proposed based on the $\mathrm{C} / \mathrm{U}$ split concept under two main scenarios of sparse and ultra-dense networks for different types of users with various QoS requirements. Key performance indicators are analysed, including coverage probability, spectral and energy efficiency. Fundamental relationships are derived between the performance and main factors, including overhead costs, SC density, transmission and circuit power, GW fronthaul, and QoS requirements. By comparing the performance under different resource management schemes, bandwidth allocation methods, and on-off approaches, the role of the satellite in future $5 \mathrm{G}$ wireless networks are summarized. Compared with current LTE networks, the proposed hybrid system can achieve nearly $40 \%$ and $80 \%$ energy efficiency in sparse and ultra-dense scenarios respectively under typical scenarios, while improving the service coverage as well in these scenarios. It can be concluded that the satellite with efficient resource management and on-off schemes can help to achieve the orchestration of the network resources, realizing higher efficiency of the whole system. Our work aims to uncover enabling research directions for the hybrid system in wireless networks. In future, the constellation of high throughput satellites in $\mathrm{Ka}$ or $\mathrm{Ku}$ band will be studied as part of the integrated $5 \mathrm{G}$ networks with millimetre waves used in dense 
small cells as a logical extension of the work provided herein.

\section{Appendix}

Proof of Theorem 2: when there are $N_{s}$ users accessing to the satellite with the available bandwidth $w_{s}$, the satellite service coverage probability can be derived:

$$
\begin{aligned}
& S_{\operatorname{cov} 2}(U)=E_{N_{s}}\left[P\left(\frac{w_{s}}{N_{s}} \log _{2}(1+S N R)>U\right)\right] \\
& =\sum_{n \geq 1} \frac{\left(\lambda_{\mathrm{su}} A_{s}\right)^{n}}{n !} e^{\left(-b \lambda_{\mathrm{su}} A_{s}\right)} P\left(\gamma>2^{\frac{U n}{w_{s}}}-1\right) \\
& =1-\sum_{n \geq 1} \frac{\left(\lambda_{\mathrm{su}} A_{s}\right)^{n}}{n !} e^{\left(-b \lambda_{\mathrm{su}} A_{s}\right)} F_{\gamma_{s d}}\left(2^{\frac{U n}{w_{s}}}-1\right),
\end{aligned}
$$

Using the expression of CDF of instant SNR of user in LMS channel in (7), it is derived:

$$
\begin{aligned}
& \bar{S}_{\operatorname{cov} 2}(U) \\
& =1-A_{0}\left(\frac{2^{\frac{U \lambda_{s u} A_{s}}{w_{s}}}-1}{\bar{\gamma}_{s d}}\right){ }_{1} F_{1}\left(m, 2, B_{0} \frac{2^{\frac{U \lambda_{s u} A_{s}}{w_{s}}}-1}{\overline{\gamma_{s d}}}\right) \\
& -\frac{A_{0} \Omega}{4 b_{0}}\left(\frac{2^{\frac{U \lambda_{s u} A_{s}}{w_{s}}}-1}{\overline{\gamma_{s d}}}\right){ }_{2}^{2} F_{2}\left(2, m ; 3,1 ; B_{0} \frac{2^{\frac{U \lambda_{s u} A_{s}}{w_{s}}}-1}{\bar{\gamma}_{s d}}\right),
\end{aligned}
$$

and the channel related parameters are introduced in Section III, finalizing the proof.

\section{REFERENCES}

[1] NSN 5G white paper, Nokia networks. http://nsn.com//file/28771/nsn-5gwhite-paper.

[2] D. Lopez-Perez, M. Ding, H. Claussen, A. H. Jafari, "Towards 1 Gbps/UE in Cellular Systems:Understanding Ultra-Dense Small Cell Deployments," IEEE Communications Surveys \& Tutorials, no. 99, pp. 1.

[3] Qualcomm Technologies, Inc, "Hyper-Dense Small Cell Deployment Trial in NASCAR Environment", April 7, 2014.

[4] I. Hwang, B. Song, S. S Soliman, "A holistic view on hyper-dense heterogeneous and small cell networks," IEEE Communications Magazine, vol. 51, no. 6, pp. 20-27, June 2013

[5] E. Oh, B. Krishnamachari, X. Liu, Z. Niu, "Toward dynamic energyefficient operation of cellular network infrastructure," IEEE Communications Magazine, vol. 49, no. 6, pp. 56-61, June 2011.

[6] C-L. I, C. Rowell, S. Han, Z. Xu, G. Li, Z. Pan, "Toward green and soft: a $5 \mathrm{G}$ perspective," IEEE Communications Magazine, vol. 52, no. 2, pp. 66-73, February 2014.

[7] Y. Cao, N. Wang, G. Kamel, Y. J. Kim, "An Electric Vehicle Charging Management Scheme Based on Publish/Subscribe Communication Framework," IEEE Systems Journal, no. 99, pp. 1-14.

[8] K. Zheng, S. Ou, J. Alonso-Zarate, M. Dohler, F. Liu, H. Zhu, "Challenges of massive access in highly dense LTE-advanced networks with machine-to-machine communications," IEEE Wireless Communications, vol. 21, no. 3, pp. 12-18, June 2014.

[9] M. Condoluci, M. Dohler, G. Araniti, A. Molinaro, K. Zheng, "Toward 5G densenets: architectural advances for effective machine-type communications over femtocells," IEEE Communications Magazine, vol. 53, no. 1, pp. 134-141, January 2015

[10] X. Zhang, J. Zhang, W. Wang, Y. Zhang, C.-L. I, Z. Pan, G. Li, Y. Chen, "Macro-assisted Data-only Carrier for 5G Green Cellular Systems," IEEE Communications Magazine, vol. 53, no. 5, pp. 223-231, May 2015.

[11] 3GPP TR 36.842 V12.0.0, Technical Specification Group Radio Access Network, "Study on Small Cell enhancements for E-UTRA and EUTRAN - Higher layer aspects," Release 12.

[12] X. Zhang, Y. Zhang, R. Yu, W. Wang, M. Guizani, "Enhancing SpectralEnergy Efficiency for LTE-Advanced Heterogeneous Networks: A Users Social Pattern Perspective,ąś IEEE Wireless Communications, vol. 21, no. 2, pp. 10-17, Apr. 2014.

[13] X. Zhang, Z. Yi, Z. Yan, G. Min, W. Wang, A. Elmokashfi, S. Maharjan, Y. Zhang, "Social Computing for Mobile Big Data," Computer, vol. 49, no. 9, pp. 86-90, Sept. 2016. doi: 10.1109/MC.2016.267.

[14] A. Capone, I. Filippini, B. Gloss, U. Barth, "Rethinking cellular system architecture for breaking current energy efficiency limits," Sustainable Internet and ICT for Sustainability (SustainIT), pp. 1-5, Oct. 2012.
[15] X. Xu, G. He, S. Zhang, Y. Chen, S. Xu, "On functionality separation for green mobile networks: concept study over LTE," IEEE Communications Magazine, vol. 51, no. 5, pp. 82-90, May 2013.

[16] B. G. Evans, "The role of satellites in 5G," 2014 7th Advanced Satellite Multimedia Systems Conference and the 13th Signal Processing for Space Communications Workshop, pp. 197-202, Sep. 2014.

[17] EC H2020 5G Infrastructure PPP Pre-structuring Model RTD \& INNO Strands, http://5g-ppp.eu.

[18] https://www.theguardian.com/technology/2015/oct/05/facebook-markzuckerberg-internet-access-africa.

[19] Y. Kawamoto, Z. Fadlullah, H. Nishiyama, N. Kato, M. Toyoshima, "Prospects and challenges of context-aware multimedia content delivery in cooperative satellite and terrestrial networks," IEEE Communications Magazine, vol. 52, no. 6, pp. 55-61, June 2014.

[20] T. Spathopoulos, O. Onireti, A.H. Khan, M. Imran, K. Arshad, "Hybrid Cognitive Satellite Terrestrial Coverage: A case study for $5 \mathrm{G}$ deployment strategies", 10th International Conference on Cognitive Radio Oriented Wireless Networks (CROWNCOM 2015), 21-23 April 2015.

[21] J. Zhang, B. Evans, M. A. Imran, X. Zhang and W. Wang, "Performance analysis of $\mathrm{C} / \mathrm{U}$ split hybrid satellite terrestrial network for $5 \mathrm{G}$ systems," 2015 IEEE 20th International Workshop on Computer Aided Modelling and Design of Communication Links and Networks (CAMAD), Guildford, 2015, pp. 97-102.

[22] J. Zhang, B. Evans, M. A. Imran, X. Zhang and W. Wang, "Green Hybrid Satellite Terrestrial Networks: Fundamental Trade-Off Analysis," 2016 IEEE 83rd Vehicular Technology Conference (VTC Spring), Nanjing, 2016, pp. 1-5.

[23] A. Laya, L. Alonso, J. Alonso-Zarate, "Is the Random Access Channel of LTE and LTE-A Suitable for M2M Communications? A Survey of Alternatives," IEEE Communications Surveys \& Tutorials, vol. 16, no. 1, pp. 4-16, 2014.

[24] J. Zhang, X. Zhang, C. Liu, W. Wang, Y. Chen, G. Li, Z. Pan, C-L. I, "Theoretical study and performance evaluation of macro-assisted data-only carrier for next generation 5G system", International Journal of Communication Systems (IJCS), 3 FEB 2015.

[25] S. Vassaki, G. T. Pitsiladis, C. Kourogiorgas, M. Poulakis, A. D Panagopoulos, G. Gardikis, S. Costicoglou, "Satellite-based sensor networks: M2M Sensor communications and connectivity analysis," 2014 International Conference on Telecommunications and Multimedia (TEMU), pp. 132-137, 28-30 July 2014.

[26] M. Sadek, S. Aissa, "Personal satellite communication: technologies and challenges," IEEE Wireless Communications, vol. 19, no. 6, pp. 28-35, December 2012

[27] J. G. Andrews, F. Baccelli, R. K. Ganti, "A Tractable Approach to Coverage and Rate in Cellular Networks," IEEE Transactions on Communications, vol. 59, no. 11, pp. 3122-3134, Nov. 2011.

[28] A. Abdi, W. C. Lau, M.-S. Alouini, M. Kaveh, "A new simple model for land mobile satellite channels: first- and second-order statistics," IEEE Transactions on Wireless Communications, vol. 2, no. 3, May 2003.

[29] S. Vassaki, A. D. Panagopoulos, P. Constantinou, "Effective Capacity and Optimal Power Allocation for Mobile Satellite Systems and Services," IEEE Communications Letters, vol. 16, no. 1, pp. 60-63, January 2012.

[30] S. Sreng, B. Escrig, M.-L. Boucheret, "Outage analysis of hybrid satelliteterrestrial cooperative network with best relay selection," Wireless Telecommunications Symposium (WTS), pp. 1-5, April 2012.

[31] O. Blume, H. Eckhardt, S. Klein, E. Kuehn, W. M. Wajda, "Energy savings in mobile networks based on adaptation to traffic statistics," Bell Labs Technical Journal, vol. 15, no. 2, pp. 77-94, Sept. 2010.

[32] G. Auer, V. Giannini, C. Desset, I. Godor, P. Skillermark, M. Olsson, M. A. Imran, D. Sabella, M. J. Gonzalez, O. Blume, A. Fehske, "How much energy is needed to run a wireless network?," IEEE Wireless Communications, vol. 18, no. 5, pp. 40-49, October 2011.

[33] J. Baliga, R. W. A. Ayre, K. Hinton, R. S. Tucker, "Green Cloud Computing: Balancing Energy in Processing, Storage, and Transport," Proceedings of the IEEE, vol. 99, no. 1, pp. 149-167, Jan. 2011.

[34] A. J. Fehske, P. Marsch, G. P. Fettweis, "Bit per Joule efficiency of cooperating base stations in cellular networks," 2010 IEEE GLOBECOM Workshops (GC Wkshps), pp. 1406-1411, Dec. 2010.

[35] J. S. Ferenc and Z. Neda, "On the size distribution of Poisson Voronoi cells." Physica A: Statistical Mechanics and its Applications, vol. 385, no. 2. pp. 518-526, 2007.

[36] S. Singh, J. G. Andrews, "Joint Resource Partitioning and Offloading in Heterogeneous Cellular Networks," IEEE Transactions on Wireless Communications, vol. 13, no. 2, pp. 888-901, February 2014.

[37] E. N. Gilbert, "Random subdivisions of space into crystals." The Annals of mathematical statistics, vol. 33, pp. 958-972, 1962.

[38] S. Singh, H. S. Dhillon, J. G. Andrews, "Offloading in Heterogeneous 
Networks: Modeling, Analysis, and Design Insights," IEEE Transactions on Wireless Communications, vol. 12, no. 5, pp. 2484-2497, May 2013.

[39] H. S. Dhillon, R. K. Ganti, F. Baccelli, J. G. Andrews, "Modeling and Analysis of K-Tier Downlink Heterogeneous Cellular Networks," IEEE Journal on Selected Areas in Communications, vol. 30, no. 3, pp. 550-560, April 2012.

[40] S. Yu, S. Kim, "Downlink capacity and base station density in cellular networks," 2013 11th International Symposium on Modeling \& Optimization in Mobile, Ad Hoc \& Wireless Networks (WiOpt), pp.119-124, May 2013.

[41] C. Qian, S. Zhang, W. Zhou, "Traffic-based dynamic beam coverage adjustment in satellite mobile communication," 2014 Sixth International Conference on Wireless Communications and Signal Processing (WCSP), pp. 1-6, Oct. 2014

[42] S. Liu, J. Wu, C. Koh, V. K. N. Lau, "A $25 \mathrm{~Gb} / \mathrm{s}(/ \mathrm{km} 2)$ urban wireless network beyond IMT-advanced," IEEE Communications Magazine, vol. 49, no. 2, pp. 122-129, February 2011.

[43] M. Z. Shafiq, L. Ji, A. X. Liu, J. Pang, J. Wang, "Large-Scale Measurement and Characterization of Cellular Machine-to-Machine Traffic," IEEE/ACM Transactions on Networking, vol. 21, no. 6, pp. 1960-1973, Dec. 2013.

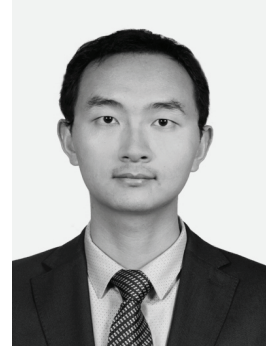

JIAXIN ZHANG (zhangjxbupt@gmail.com) received the B.E degree in information engineering 2012 from Beijing University of Posts and Telecommunications, Beijing, China, where he is currently pursuing the Ph.D. degree in the Key Laboratory of Universal Wireless Communications, School of Information and Communication Engineering. He is also with the Institute for Communication Systems (ICS), University of Surrey, Guildford, UK. His research interests are mainly wireless communications and networks, green communication, small cell enhancement technologies and 5G network architecture.

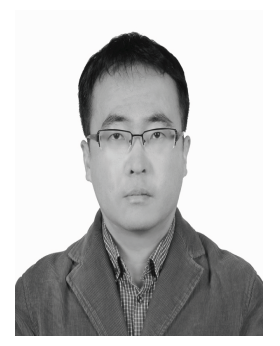

Xing Zhang (zhangx @ ieee.org) is Full Professor with the School of Information and Communications Engineering, Beijing University of Posts and Telecommunications (BUPT), China. His research interests are mainly in $5 \mathrm{G}$ wireless communications and networks, green communications, cognitive radio and cooperative communications, big data and Internet of Things. $\mathrm{He}$ is the author/coauthor of two technical books and more than 100 papers in top journals and international conferences and filed more than 30 patents. Prof. Zhang has served as General Co-Chairs of the 3rd IEEE International Conference on Smart Data (SmartData-2017), as a TPC Cochair/TPC member for a number of major international conferences, including MobiQuitous 2012, IEEE ICC/GLOBECOM/WCNC, CROWNCOM, Chinacom, etc. He received the Best Paper Awards in the 9th International Conference on Communications and Networking in China (Chinacom 2014), the 17th International Symposium on Wireless Personal Multimedia Communications (WPMC 2014), and the 8th IEEE International Conference on Wireless Communications and Signal Processing (IEEE WCSP 2016). He is a Senior Member of IEEE and IEEE ComSoc, Member of CCF.

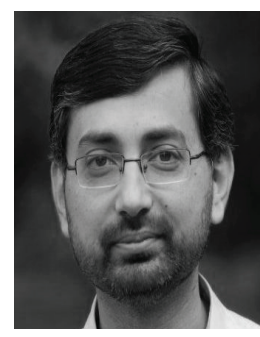

\section{Muhammad Ali Imran}

(muhammad.Imran@glasgow.ac.uk) received his M.Sc (Distinction) and Ph.D. degrees from Imperial College London, UK, in 2002 and 2007, respectively. He is a Professor in Communication Systems in University of Glasgow, Vice Dean of Glasgow College UESTC and Program Director of Electrical and Electronics with Communications. He is an adjunct Professor at the University of Oklahoma, USA and a visiting Professor at the Institute for Communication Systems -home of $5 \mathrm{G}$ Innovation Centre. He has led a number of multimillion-funded international research projects encompassing the areas of energy efficiency, fundamental performance limits, sensor networks and selforganising cellular networks. He is also leading the new physical layer work area for $5 \mathrm{G}$ innovation centre at Surrey. He has a global collaborative research network spanning both academia and key industrial players in the field of wireless communications. He has supervised 21 successful $\mathrm{PhD}$ graduates and published over 200 peer-reviewed research papers including more than 20 IEEE Transaction papers. He secured first rank in his B.Sc. and a distinction in his M.Sc. degree along with an award of excellence in recognition of his academic achievements conferred by the President of Pakistan. He has been awarded IEEE Comsocaŕs Fred Ellersick award 2014 and FEPS Learning and Teaching award 2014 and twice nominated for Tony Jeanaŕs Inspirational Teaching award. He is a shortlisted finalist for The Wharton-QS Stars Awards 2014 for innovative teaching and VCąís learning and teaching award in University of Surrey. He is a senior member of IEEE and a Senior Fellow of Higher Education Academy (SFHEA), UK

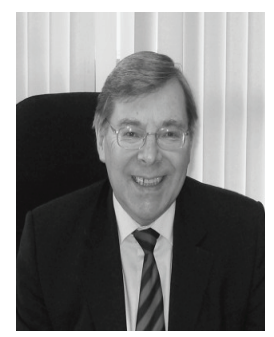

Barry G. Evans (b.evans@ surrey.ac.uk) Professor Evans has $\mathrm{BSC}$ and $\mathrm{PhD}$ degrees from the University of Leeds, is a Fellow of the UK Royal Academy of Engineering and of the IET and senior member of IEEE and AIAA. From 1968 to 83 he was British Telecom lecturer to Reader at the University of Essex in Telecommunication systems. He was appointed to the Alec Harley Reeves chair of Information systems engineering at the University of Surrey in 1983 and was founder Director of the Centre for satellite engineering research and then the Centre for Communication Systems Research. He was Dean of Engineering 99-01 and Pro-Vice Chancellor for research and Enterprise from 01-09. Barry Evans has researched in satellite communications, radio propagation, signal processing and networking and has over 600 publications in the literature plus 3 books. He is Editor of the International Journal of Satellite communications.

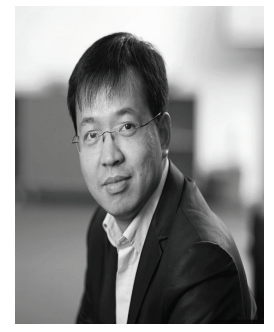

Yan Zhang(yanzhang@ieee.org) is Full Professor at the Department of Informatics, University of Oslo, Norway. He received a $\mathrm{PhD}$ degree in School of Electrical \& Electronics Engineering, Nanyang Technological University, Singapore. He is an Associate Technical Editor of IEEE Communications Magazine, an Editor of IEEE Transactions on Green Communications and Networking, an Editor of IEEE Communications Surveys \& Tutorials, an Editor of IEEE Internet of Things Journal, and an Associate Editor of IEEE Access. He serves as chair positions in a number of conferences, including IEEE GLOBECOM 2017, IEEE VTC-Spring 2017, IEEE PIMRC 2016, IEEE CloudCom 2016, IEEE ICCC 2016, IEEE CCNC 2016, IEEE SmartGridComm 2015, and IEEE CloudCom 2015. He serves as T$\mathrm{PC}$ member for numerous international conference including IEEE INFOCOM, IEEE ICC, IEEE GLOBECOM, and IEEE WCNC. His current research interests include: next-generation wireless networks leading to $5 \mathrm{G}$, green and secure cyber-physical systems (e.g., smart grid, healthcare, and transport). He is IEEE VTS (Vehicular Technology Society) Distinguished Lecturer. He is also a senior member of IEEE, IEEE ComSoc, IEEE CS, IEEE PES, and IEEE VT society. He is a Fellow of IET.

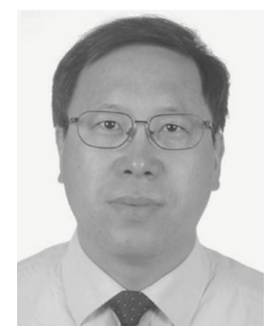

Wenbo Wang (wbwang@ @upt.edu.cn) received B.S., M.S., and Ph.D. degrees from BUPT in 1986, 1989, and 1992, respectively. He is currently a professor and executive vice dean of the Graduate School, BUPT. Currently, he is the assistant director of the Key Laboratory of Universal Wireless Communication, Ministry of Education. He has published more than 200 journal and international conference papers, and six books. His current research interests include radio transmission technology, wireless network theory, and software radio technology. 